\title{
A Parametrization-Invariant Fourier Approach to Planar Linkage Synthesis for Path Generation
}

\author{
Xiangyun $\mathrm{Li}^{1,2}$ and Peng Chen ${ }^{1}$ \\ ${ }^{1}$ School of Mechanical Engineering, Southwest Jiaotong University, Chengdu 610031, China \\ ${ }^{2}$ Sichuan Provincial Machinery Research \& Design Institute, Chengdu 610063, China \\ Correspondence should be addressed to Peng Chen; chenpeng@swjtu.edu.cn
}

Received 8 May 2017; Revised 16 September 2017; Accepted 28 September 2017; Published 7 November 2017

Academic Editor: Kalyana C. Veluvolu

Copyright (c) 2017 Xiangyun Li and Peng Chen. This is an open access article distributed under the Creative Commons Attribution License, which permits unrestricted use, distribution, and reproduction in any medium, provided the original work is properly cited.

\begin{abstract}
This paper deals with the classic problem of the synthesis of planar linkages for path generation. Based on the Fourier theory, the task curve and the synthesized four-bar coupler curve are regarded as the same curve if their Fourier descriptors match. Using Fourier analysis, a curve must be given as a function of time, termed a parametrization. In practical applications, different parametrizations can be associated with the same task and coupler curve, respectively; however, these parametrizations are Fourier analyzed to different Fourier descriptors, thus resulting in the mismatch of the task and coupler curve. In this paper, we present a parametrization-invariant method to eliminate the influence of parametrization on the values of Fourier descriptors by unifying given parametrizations to the arc length parametrization; meanwhile, a new design space decoupling scheme is introduced to separate the shape, size, orientation, and location matching of the task and four-bar curve, which leads naturally to an efficient synthesis approach.
\end{abstract}

\section{Introduction}

This paper studies the problem of dimensional synthesis of planar four-bar linkages for path generation. In the field of computational shape analysis [1], it is routine to process and simplify shapes before the comparison. This simplified representation of shapes is called shape descriptor or shape signature. Of our interest is the use of Fourier descriptor (FD) in characterizing the coupler path of four-bar mechanisms.

The application of FD to mechanism design was first explored by Freudenstein [2] in the context of function generation. The research was followed by Funabashi and Freudenstein [3], Farhang et al. [4, 5], Chu and Cao [6], McGarva and Mullineux [7], McGarva [8], Ullah and Kota [9], and Nie and Krovi [10]. Lately, Chu and Sun [11, 12] and Sun and Chu [13] have extended FD based method to the synthesis of spherical and spatial linkages. One of the key features of the FD based method is the ability to decouple the nine design variables involved in path generation. Ullah and Kota [9] were the first to present this conclusion and used it to reduce the dimension of the search space from nine to five.
Recently, Wu et al. [14] further reduced the search dimension from five to four. Another important feature is that while the path of a coupler point depends on the choice of the coupler point, one may extract a subset of FDs of the path in such a way that they depend only on the linkage dimensions but not on the choice of the coupler point. This means that, for each four-bar linkage, one set of Fourier descriptors can be used to tag all its coupler curves. Chu et al. [15] made this key observation and achieved significant reduction in the size of the database for numerical atlas. Xie and Chen [16] were the first to extend Fourier descriptor method to the image space of kinematic mapping to solve the whole cycle motion generation problem in four-bar linkage synthesis.

While proven to be an effective tool for the path and motion generation problems, the FD based method in its current form, however, is not without limitations. The prerequisite for applying the Fourier transform is that the path should be mathematically defined as a function of time $t$, also known as parametric representation or parametrization. Given the parametrization of the path, FDs for the path are then obtained through the Fourier transform and used 
to identify or tag the path. However, numerous kinds of parametrizations can be introduced to define the same path [17], and the Fourier descriptors for each parametrization are different, indicating that the same path can have different identities or tags. In terms of the path generation problem, the objective is to match the FDs of the task and four-bar paths. The parametrization of the task path is prescribed according to the user's need or practical applications, while the parametrization of the synthesized four-bar path, in traditional Fourier approaches, is generated by rotating the input link of the four-bar mechanism at a constant angular speed. In the case that the desired path is actually the fourbar path but has the parametrization equivalent to the one generated by the input link rotating at a nonuniform angular speed, their FDs will differ from each other. Therefore, in order to better match the task path with the four-bar path, we need to compare their FDs under the same parametrization.

In this paper, we use arc length parametrization to be the parametrization for the task and four-bar coupler curve. Arc length parametrization is based on an intrinsic property of curve: arc length. Before matching two paths by their FDs, we need to reparameterize them using arc length as the parameter. However, direct comparison between the synthesized and desired path under the arc length parametrization requires the search for ten design variables of the four-bar mechanism simultaneously, which would cause tremendous computational cost. Considering that a curve is characterized by its location, orientation, size, and shape, we present a novel scheme for decoupling the design space of four-bar mechanisms by identifying the design variables that separately affect the location, orientation, size, and shape of a four-bar path. Instead of matching the entire task and fourbar path, we can first match their shapes to obtain a subset of design variables and then match their sizes, orientations, and locations, respectively, to determine the remaining variables.

In order to make the shape comparison, the curve normalization procedure proposed by Dikabar and Mruthyunjaya [18] is used to remove the location, orientation, and size information of a path. This procedure treats the path as a polygonal curve and uses its vertices' coordinates to normalize the polygonal curve to a canonical curve within a bounding unit rectangle. Following the normalization step, we apply arc length parametrization to the normalized task and four-bar path so as to compare their FDs against the same parametrization. For efficiently searching the shape-related design variables that generate the shape of four-bar path with the best-match FDs, an artificial neural network (ANN) is trained to establish the relationship between those design variables and their corresponding FDs. As for the finding of remaining variables, we formulate a restoring method to determine their values, respectively, through size, orientation, and location matching.

One issue worth attention is that the path is actually defined by an ordered sequence of points uniformly sampled in the time domain; after reparametrizing the path using arc length, the parameter values (arc length values) associated with the sampling points could be unevenly distributed. In this case, the discrete Fourier transform (DFT) is not applicable; instead, the trapezium rule presented by Vasiliu

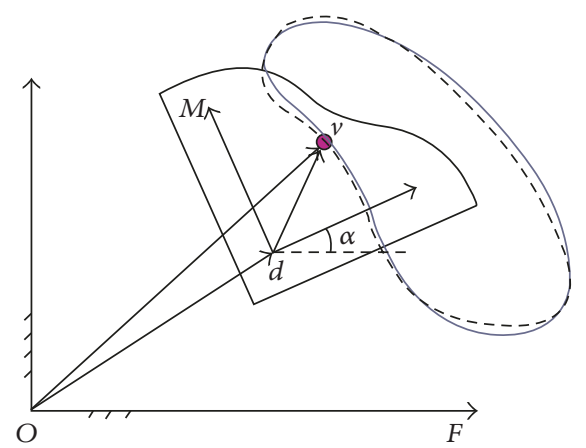

FIgURE 1: $F$ is the fixed frame and $M$ the moving frame. Dashed-line path is generated by the point $v$ on the rigid body and solid-line path synthesized by linkage.

and Yannou [19] or the least squares curve fitting approach by $\mathrm{Wu}$ et al. [20] can be used to compute the Fourier descriptors.

The organization of the paper is as follows. Section 2 reviews how FDs are used for representing a closed curve on the plane and discuss the issue of parametrization in detail. Section 3 presents the loop closure equation of a fourbar linkage in a form that is suitable for the development of this paper and its Fourier analysis. Section 4 introduces how to decouple the design variables in view of the issue of parametrization. Section 5 illustrates the numerical synthesis algorithm, involving curve normalization, arc length parametrization, backpropagation artificial neural network, and restoring method. Section 6 gives results and discussion of our approach to justify its effectiveness in path generation, with comparison against traditional Fourier based path generation algorithms.

\section{Parametrization Dependence of Fourier Based Path Representation}

This section reviews the use of FD in characterizing a path generated by a point on a rigid body and discusses the issue of parametrization.

A planar rigid body is shown in Figure 1. The position of the moving body relative to a fixed frame $F$ is represented by a frame $M$ attached to the moving body. Assume that the rigid body is moving in the plane; one point $v$ traces out the closed curve (task curve) marked with dashed line while the one with solid line is the matching curve to be synthesized by a four-bar mechanism.

When a closed task curve is specified as a periodic complex function $z(t)=x(t)+j y(t)$, it can be approximated by a finite series of sinusoidal functions in complex form as

$$
z(t) \approx \sum_{k=-p}^{+p} \alpha_{k} e^{j k \omega t}
$$

where $p$ is a small positive integer defining the maximum order of the harmonic terms used for the approximation and the coefficients $\alpha_{k}$ are termed the FDs of the given path. Given 
the cycle time is 1 , that is, $\omega=2 \pi$, the FDs $\alpha_{k}$ are calculated as

$$
\alpha_{k}=\int_{0}^{1} e^{-j k 2 \pi t} z(t) d t
$$

Equations (1) and (2) are established on the path function $z(t)$, also known as the parametric form of the path. However, various parametrizations can be used to define a path of the same geometry [17]. For example, the unit circle can be represented either as the standard parametric form

$$
z_{1}(t)=e^{j 2 \pi t}
$$

or as an alternative form

$$
z_{2}(t)=e^{j 2 \pi\left(0.4 t^{3}+0.5 t^{2}+0.1 t\right)}
$$

The unit circle with the two parametrizations is shown in Figure 2. The effect of changing the parametrization is equivalent to altering the speed at which the path is traced out. The parametrization $z_{1}(t)$ can be viewed as being traced by a point revolving around the origin at a constant angular speed of $2 \pi$ while the parametrization $z_{1}(t)$ is viewed as being traced by a point revolving around the origin at a varying angular speed of $2 \pi\left(1.2 t^{2}+t+0.1\right)$. In other words, a point will reach a certain position on the unit circle using different time for these two parametrizations.

Now, we obtain the Fourier representation of these two parametrizations. The Fourier series approximation of $z_{1}(t)$ is given by itself:

$$
z_{1}(t)=e^{j 2 \pi t}
$$

On the other hand, the Fourier expansion of $z_{2}(t)=$ $\cos \left[2 \pi\left(0.4 t^{3}+0.5 t^{2}+0.1 t\right)\right]+j \sin \left[2 \pi\left(0.4 t^{3}+0.5 t^{2}+0.1 t\right)\right]$ is given by

$$
\begin{aligned}
z_{2}(t)= & (0.2604+0.2159 j) \\
& +(0.0179+0.0956 j) e^{-j 2 \pi t} \\
& +(0.3415-0.7980 j) e^{j 2 \pi t} \\
& +(0.0020+0.0413 j) e^{-j 4 \pi t} \\
& +(0.3018+0.1088 j) e^{j 4 \pi t} \\
& +(0.0005+0.0237 j) e^{-j 6 \pi t} \\
& +(0.0578+0.0981 j) e^{j 6 \pi t} .
\end{aligned}
$$

It is noted that the FDs of these two parametrizations disagree with each other, yet they both define the unit circle. Suppose that the unit circle is the task path where we require a crank-rocker mechanism as shown in Figure 3 to generate. The only candidate capable of exactly tracing the unit circle is the moving pivot $A$, which can be regarded as extreme case of coupler point $P$. Traditional Fourier approaches assume that the input link $A_{0} A$ rotates at constant angular speed $\omega=2 \pi$. If the parametrization of the unit circle takes the standard form $z_{1}(t), A$ will be found as the solution; however, if the parametrization changes to $z_{2}(t)$, a different coupler curve other than $A$ will be found that can only approximate rather than exactly match the unit circle.

When the task curve is represented by a discrete sequence of points $P_{i}(i=0, \ldots, n-1)$, the discrete Fourier transform (DFT) is used to obtain the FDs. The inverse DFT of $P_{i}$ is represented as

$$
P_{i}=\sum_{k=0}^{N-1} \alpha_{k} e^{j k(2 \pi i / N)} .
$$

The FDs $\alpha_{k}$ are given by forward DFT:

$$
\alpha_{k}=\frac{1}{N} \sum_{i=0}^{N-1} P_{i} e^{-j k(2 \pi i / N)} .
$$

The validity of (7) and (8) is based on the assumption that $P_{i}$ are associated with equally spaced values of the parameter $t$, that is, $t=0,1 / n, 2 / n, \ldots,(n-1) / n$. If a different parametrization is assigned to $P_{i}, P_{i}$ will be denoted as $P_{i}\left(t_{i}\right)$ in which $t_{i}$ is an unequally distributed sequence in $[0,1]$. In this case, (8) is not applicable. In order to address the nonuniform spacing of $t$, we apply the curve fitting approach to obtain the uniform FDs, which is formulated in the least squares sense by defining and minimizing the following error function:

$$
\Delta=\sum_{i=1}^{n}\left|P_{i}\left(t_{i}\right)-\sum_{k=-p}^{+p} \alpha_{k} e^{j k 2 \pi t_{i}}\right|^{2},
$$

where $|\cdot|$ denotes the magnitude of a complex number. Note that (9) is also suitable for the uniform spacing case.

For the same sequence of points $P_{i}$ with two different parametrizations $t_{i}$ and $t_{i}^{\prime}$, the FDs will be different, as in (9). $P_{i}\left(t_{i}\right)=P_{i}\left(t_{i}^{\prime}\right)$ for all $i=0,1, \ldots, n-1$ while $e^{j k 2 \pi t_{i}}$ is different from $e^{j k 2 \pi t_{i}^{\prime}}$. Therefore, the Fourier representation of a task curve, given either as a continuous function or as a sequence of points, is dependent on the underlying parametrization. In what follows, we present the Fourier analysis of the four-bar mechanism with the parametrization issue considered.

\section{Fourier Analysis of the Four-Bar Mechanism}

Consider the planar four-bar linkage shown in Figure 3 with $X O Y$ being the fixed coordinate frame. The fixed pivot $A_{0}$ is located at point $\left(x_{0}, y_{0}\right)$ with $A_{0} B_{0}$ being the ground link and $A_{0} A$ the input link. Let $l_{i}$ denote the length of the $i$ th link and $\theta_{i}$ the angle measured from $x$-axis of the fixed frame. Let $\phi, \lambda$, and $\psi$ be the angles of links $A_{0} A, A B$, and $B_{0} B$ as measured from the ground link $A_{0} B_{0}$, respectively.

In consideration of the parametrization issue discussed in Section 2, the input link is specified to rotate at a varying angular speed of $\omega(t)$, and therefore the rotation angle function of the input link is given as

$$
\phi(t)=\int_{0}^{t} \omega(\tau) d \tau+\phi_{0} \quad t \in[0,1],
$$

where $\phi_{0}$ is the initial input angle and $\int_{0}^{1} \omega(\tau) d \tau=2 \pi$. 


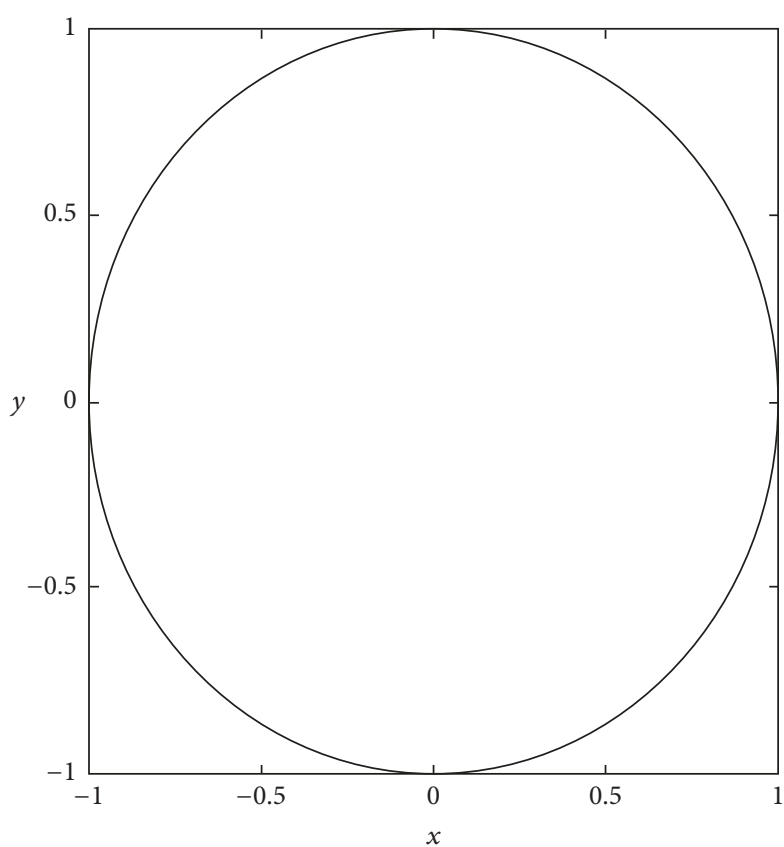

(a)

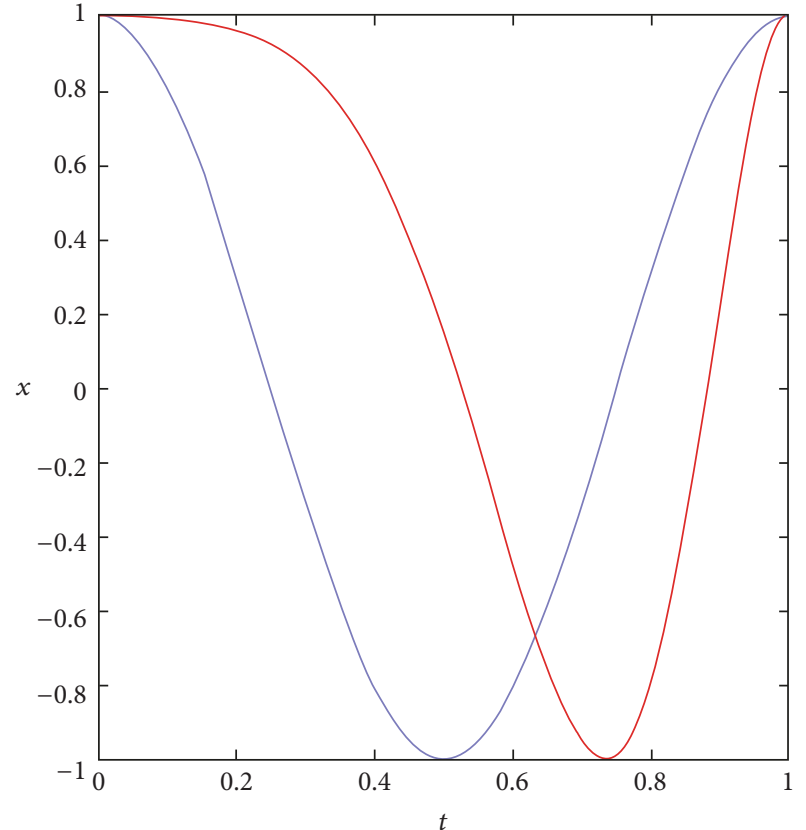

(b)

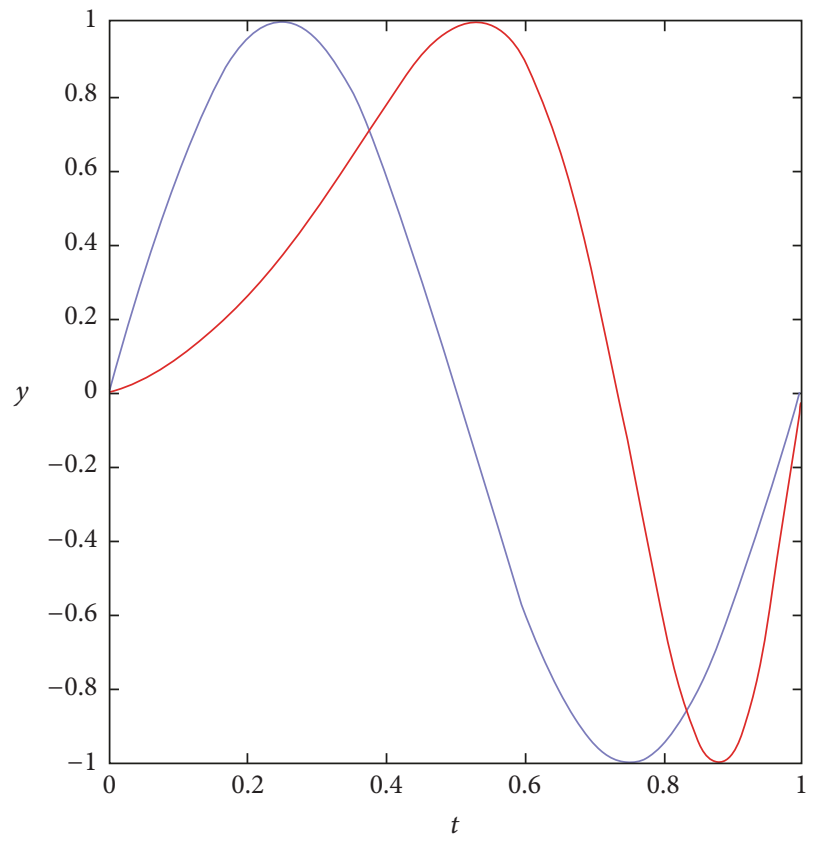

(c)

Figure 2: (a) The unit circle. (b) The $x$-axis components of two parametrizations, respectively, where the blue line is the graph for the $x$-axis component $\cos (2 \pi t)$ of $z_{1}(t)=\cos (2 \pi t)+j \sin (2 \pi t)$, and the red line is the graph for the $x$-axis component $\cos \left[2 \pi\left(0.4 t^{3}+0.5 t^{2}+0.1 t\right)\right]$ of $z_{2}(t)=\cos \left[2 \pi\left(0.4 t^{3}+0.5 t^{2}+0.1 t\right)\right]+j \sin \left[2 \pi\left(0.4 t^{3}+0.5 t^{2}+0.1 t\right)\right]$. (c) The $y$-axis components of two parametrizations, respectively, that is, $\sin (2 \pi t)$ and $\sin \left[2 \pi\left(0.4 t^{3}+0.5 t^{2}+0.1 t\right)\right]$. by

Using loop closure equations, the coupler angle $\lambda$ is given

$$
e^{j \lambda}=\frac{-B(\phi) \pm \sqrt{\Delta_{1}(\phi) \Delta_{2}(\phi)}}{2 A(\phi)},
$$

where

$$
\begin{aligned}
& l_{21}=\frac{l_{2}}{l_{1}}, \\
& l_{31}=\frac{l_{3}}{l_{1}},
\end{aligned}
$$




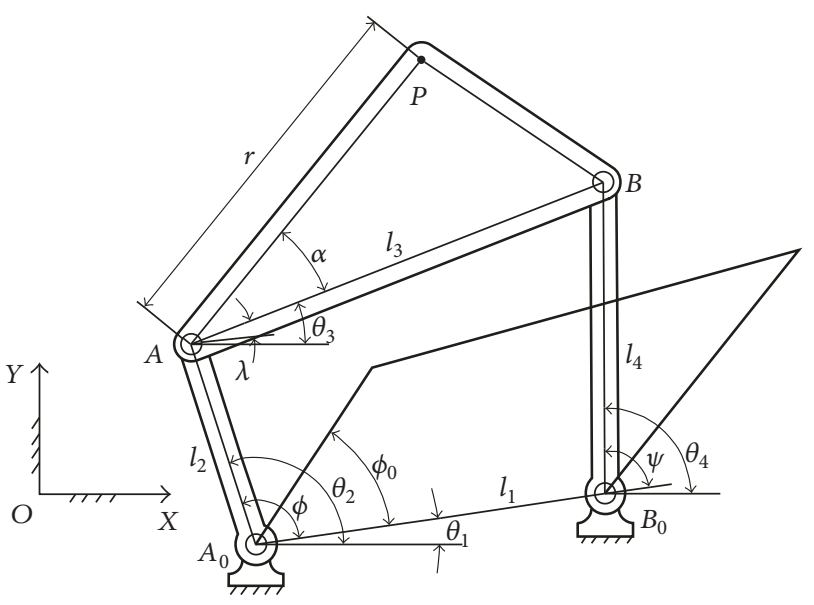

Figure 3: A four-bar mechanism.

$$
\begin{aligned}
l_{41} & =\frac{l_{4}}{l_{1}} \\
A(\phi) & =l_{31}\left(l_{21} e^{-j \phi}-1\right), \\
B(\phi) & =1+l_{21}^{2}+l_{31}^{2}-l_{41}^{2}-2 l_{21} \cos \phi, \\
\Delta_{1}(\phi) & =1+l_{21}^{2}-\left(l_{31}+l_{41}\right)^{2}-2 l_{21} \cos \phi, \\
\Delta_{2}(\phi) & =1+l_{21}^{2}-\left(l_{31}-l_{41}\right)^{2}-2 l_{21} \cos \phi
\end{aligned}
$$

and the sign \pm corresponds to the two configurations of the four-bar linkage for each input angle.

In view of (11), it is clear that the complex exponential of coupler angle $\lambda$ depends only on the input angle $\phi$ as well as three link ratios. Also, (11) leads to the following well-known feasibility condition:

$$
\Delta_{1}(\phi) \Delta_{2}(\phi) \leq 0
$$

The input link is crank if this inequality holds for all $\phi \epsilon$ $[0,2 \pi]$; otherwise, it is a rocker.

Now let us consider Fourier representation of the coupler curve of a four-bar mechanism. Let $\mathbf{A}_{0}=x_{0}+j y_{0}$ be the complex number specifying the fixed pivot $A_{0}$ and let $\mathbf{z}_{c}=x_{c}+j y_{c}=r e^{j \alpha}$ represent the location of coupler point $P$ relative to the coupler link $A B$. The complex-form coordinates of the coupler point relative to global frame $X O Y$ can be represented as

$$
\mathbf{P}=\mathbf{A}_{0}+l_{2} e^{j \theta_{2}}+\mathbf{z}_{c} e^{i \theta_{3}}=\mathbf{A}_{0}+l_{2} e^{j \theta_{1}} e^{j \phi}+\mathbf{z}_{c} e^{j \theta_{1}} e^{j \lambda} .
$$

Note that ten design variables $\left\{l_{1}, l_{2}, l_{3}, l_{4}, x_{0}, y_{0}, \theta_{1}, r, \alpha, \phi_{0}\right\}$ are involved in (14).
In view of (10) and (11), the Fourier representations of $e^{j \phi}$ and $e^{j \lambda}$ are given as

$$
\begin{aligned}
e^{j \phi} & =\sum_{k=-\infty}^{\infty} C_{k} e^{j \phi_{0}} e^{j k 2 \pi t}, \\
e^{j \lambda} & =\sum_{k=-\infty}^{\infty} D_{k} e^{j k 2 \pi t} .
\end{aligned}
$$

Substituting (15) and (16) into (14), we obtain the Fourier representation of the coupler path $\mathbf{P}$ :

$$
\mathbf{P}=\sum_{k=-\infty}^{\infty} \beta_{k} e^{i k 2 \pi t},
$$

where

$$
\begin{aligned}
& \beta_{0}=\mathbf{A}_{0}+l_{2} C_{0} e^{j\left(\theta_{1}+\phi_{0}\right)}+\mathbf{z}_{c} e^{j \theta_{1}} D_{0}, \\
& \beta_{k}=\left.\left(l_{2} C_{k} e^{j \phi_{0}}+\mathbf{z}_{c} D_{k}\right) e^{j \theta_{1}}\right|_{k \neq 0} .
\end{aligned}
$$

By observing (18), it can be concluded that the FDs $\beta_{k}(k \neq 0)$ are determined by $\left\{l_{2}, r, \alpha, \phi_{0}, \theta_{1}, C_{k}, D_{k}\right\}$, of which $C_{k}$ depend on the variable part of the rotation function $\phi(t)-\phi_{0}$, while $D_{k}$ are determined by $\phi(t)$ and $\left\{l_{21}, l_{31}, l_{41}\right\}$. Furthermore, $\phi(t)$ is related to the parametrization of $\mathbf{P}$, that is, the speed at which $\mathbf{P}$ is traced out. $\beta_{0}$ is determined by all the aforementioned factors plus the location of the fixed pivot $A_{0}$.

In order to match or approximate a task path as closely as possible, we seek to match its FDs denoted as $\alpha_{k}$ with four-bar curve FDs $\beta_{k}$, both of which are computed against the arc length parametrization. First, eight design variables $\left\{l_{2}, r, \alpha, \phi_{0}, \theta_{1}, l_{21}, l_{31}, l_{41}\right\}$ are used to match $\alpha_{k}$ and $\beta_{k}(k \neq$ $0)$; second, the rest of two variables $x_{0}$ and $y_{0}$ are used to match $\alpha_{0}$ and $\beta_{0}$. The main disadvantage of this direct comparison approach is that we have to search the eight design variables involved in the first step simultaneously at a high computation cost. From a geometric point of view, a path is characterized by its location, orientation, size, and shape. In Section 4, a novel decoupling scheme will be presented to classify the design parameters in terms of their roles in determining the location, orientation, size, and shape of the four-bar curve.

\section{Decoupling of Design Variables}

In this section, we seek to decouple the design space of a fourbar mechanism by identifying the relationship between the design variables and the geometry of a four-bar coupler curve.

Before the introduction of our method, we briefly review the decoupling scheme in traditional Fourier based path synthesis approaches. The assumption made in traditional approaches that the input link rotates with a constant speed can lead to a simpler decomposition scheme. First, (10) is reduced to

$$
\phi(t)=\omega t+\phi_{0}=2 \pi t+\phi_{0} .
$$


Second, (15) is simplified to

$$
e^{j \phi}=e^{j \phi_{0}} e^{j k 2 \pi t}
$$

Hence, (18) becomes

$$
\begin{aligned}
& \beta_{0}=\mathbf{A}_{0}+\mathbf{z}_{c} e^{j \theta_{1}} D_{0}, \\
& \beta_{1}=\left(l_{2} e^{j \phi_{0}}+\mathbf{z}_{c} D_{1}\right) e^{j \theta_{1}}, \\
& \beta_{k}=\left.\mathbf{z}_{c} D_{k} e^{j \theta_{1}}\right|_{k \neq 0,1} .
\end{aligned}
$$

By observing (21), $\beta_{k}(k \neq 0,1)$ depend on $\left\{l_{21}, l_{31}, l_{41}, r, \alpha\right.$, $\left.\phi_{0}, \theta_{1}\right\}$. In the method presented by Chu et al. [15], further decoupling is carried out by noting that $\beta_{k} / \beta_{-1}(k \neq 0,1)$ is decided only by $\left\{l_{21}, l_{31}, l_{41}, \phi_{0}\right\}$. In the method of Wu et al. [20], a least squares method is formulated to separate $\left\{l_{21}, l_{31}, l_{41}, \phi_{0}\right\}$ from $\left\{l_{21}, l_{31}, l_{41}, r, \alpha, \phi_{0}, \theta_{1}\right\}$.

Now with the additional complexity of parametrization issue, we need to adopt a different approach to design space decoupling. As stated above, ten design parameters $\left\{l_{1}, l_{2}, l_{3}, l_{4}, x_{0}, y_{0}, \theta_{1}, r, \alpha, \phi_{0}\right\}$ are involved in the synthesis process. Through the following derivation, we will see their different roles in determining the location, orientation, size, and shape of the coupler curve.

The point coordinates of the coupler curve are captured by (14), which can be divided into two parts:

$$
\mathbf{P}=\mathbf{P}_{1}+\mathbf{P}_{2} e^{j \theta_{1}}
$$

where

$$
\begin{aligned}
& \mathbf{P}_{1}=\mathbf{A}_{0}=x_{0}+j y_{0}, \\
& \mathbf{P}_{2}=l_{2} e^{j \phi}+\mathbf{z}_{c} e^{j \lambda} .
\end{aligned}
$$

By examining the above equations, (22) and (23), it is first noted that $\mathbf{P}_{1}$ only affects the location of the coupler curve because it translates the point coordinates of the entire curve by a vector of $\left(x_{0}, y_{0}\right)$. Hence, the curve represented by $\mathbf{P}_{2} e^{j \theta_{1}}$ has the same orientation, size, and shape as the original coupler curve $\mathbf{P}$. Second, $e^{j \theta_{1}}$ only influences the orientation of the four-bar curve as it rotates the entire curve by an angle of $\theta_{1}$. Therefore, the curve described by $\mathbf{P}_{2}$ has the same size and shape as $\mathbf{P}$. Furthermore, we divide $\mathbf{P}_{2}$ by $l_{1}$ to get

$$
\overline{\mathbf{P}}_{2}=\frac{\mathbf{P}_{2}}{l_{1}}=\frac{l_{2}}{l_{1} e^{j \phi}}+\frac{\mathbf{z}_{c}}{l_{1} e^{j \lambda}} .
$$

The curve described by $\overline{\mathbf{P}}_{2}$ has the same shape as the four-bar curve $\mathbf{P}$, but with $1 / l_{1}$ times the size of $\mathbf{P}$.

Until now, we have isolated $\left\{x_{0}, y_{0}\right\}$ as location-related variables, $\left\{\theta_{1}\right\}$ as orientation-related variable, and $\left\{l_{1}\right\}$ as sizerelated variable. Since the curve $\overline{\mathbf{P}}_{2}$ is of the same shape as original coupler curve $\mathbf{P}$, the variables contained in $\overline{\mathbf{P}}_{2}$ will influence the shape of the coupler curve. In view of (11) and (12) and $\mathbf{z}_{c}=x_{c}+j y_{c}=r e^{j \alpha}$, those variables are $\left\{l_{21}, l_{31}, l_{41}, x_{c} / l_{2}, y_{c} / l_{2}\right\}$ (note here that $\phi_{0}$ decides only the starting point to trace out the coupler curve and does not affect the geometry at all). In addition, to represent the coupler point coordinate $\left(x_{c}, y_{c}\right)$ relative to the coupler link $l_{3}$ instead of ground link $l_{1},\left\{x_{c} / l_{1}, y_{c} / l_{1}\right\}$ are changed to $\left\{x_{c} / l_{3}, y_{c} / l_{3}\right\}$, and finally the five shape-related variables become

$$
\left\{l_{21}, l_{31}, l_{41}, \frac{x_{c}}{l_{3}}, \frac{y_{c}}{l_{3}}\right\} .
$$

Through the above analysis, we have identified the design variables in (25) as shape-related variables, which reflect the link ratios and the relative coupler point coordinates to the coupler coordinate frame. Now, the procedure of matching task and four-bar path is greatly simplified due to the new decoupling scheme. Instead of matching the FDs of the entire task path and the entire four-bar curve, we only need to match the FDs of their shapes, by searching the values of the design variables in (25). Following the shape match, subsequent steps of size, orientation, and location match can determine the values of $l_{1}, \theta_{1}, x_{0}$, and $y_{0}$.

\section{Numerical Synthesis}

In this section, we first present the shape match algorithm to find the design parameters in (25). In order for the shape comparison to be independent of path location, orientation, and size, a curve normalization procedure must be carried out to transform the task and the curve $\overline{\mathbf{P}}_{2}$, which is the shape equivalence of the original four-bar curve $\mathbf{P}$, into their canonical configurations, respectively. Moreover, previous to matching the FDs of the normalized task and four-bar path, we need to convert their parametrizations into arc length parametrizations, respectively. To enhance the efficiency of shape match, we employ artificial neural networks to establish the relationship between the parameters in (25) and the FDs of the normalized four-bar path. After the shape match, we introduce the restoring method to implement the size, orientation, and location match for the sake of finding the remaining variables.

5.1. Curve Normalization. Curve normalization procedure (see Appendix), as shown in Figure 4, was first proposed by Dikabar and Mruthyunjaya [18] and later adopted by Marín and González [21]. Recently, Galán-Marín et al. [22] applied it with wavelet descriptors approach to efficiently synthesize crank-rocker mechanisms constrained by optimal transmission angle. The principle of this procedure is that we treat a closed curve as a closed polygon of $N$ vertices and $N$ sides, with the $N$ vertices corresponding to the $N$ points of the discretized curve. Through curve normalization, the major principal axis of moment of inertia is aligned with the $x$-axis; then, the width $w$ and height $h$ of the bounding box of the curve are evaluated; finally, the curve with the bounding box is scaled by a factor of $1 / w$ and translated to the origin. The normalized curve preserves only the shape of the original curve. Note that the direction of the curve, either clockwise or counterclockwise, should also be normalized, which can be implemented using the shoelace formula. 


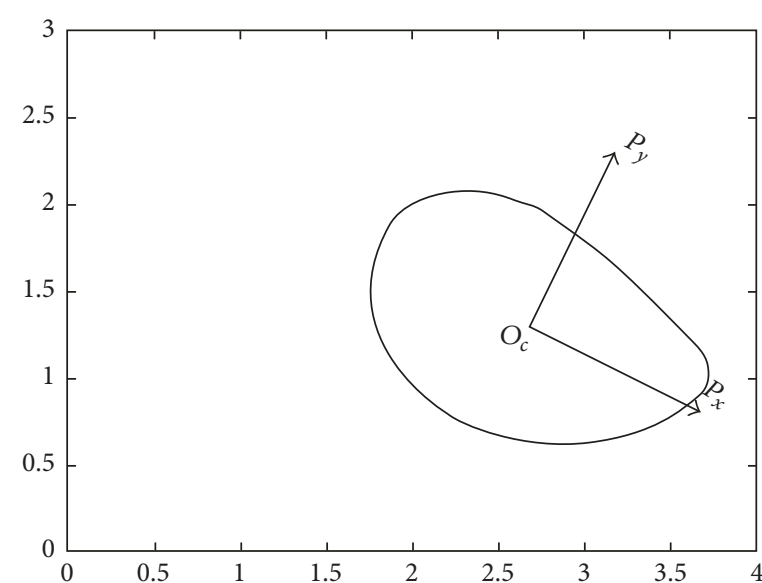

(a)

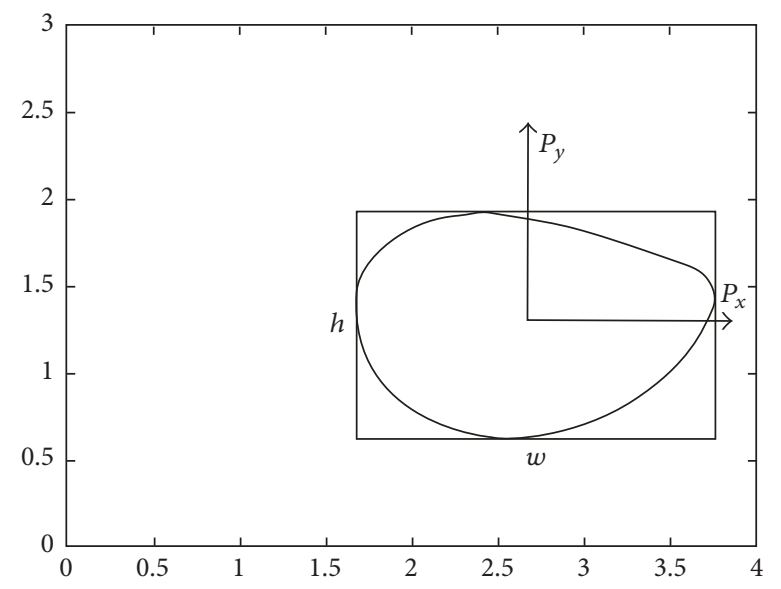

(c)

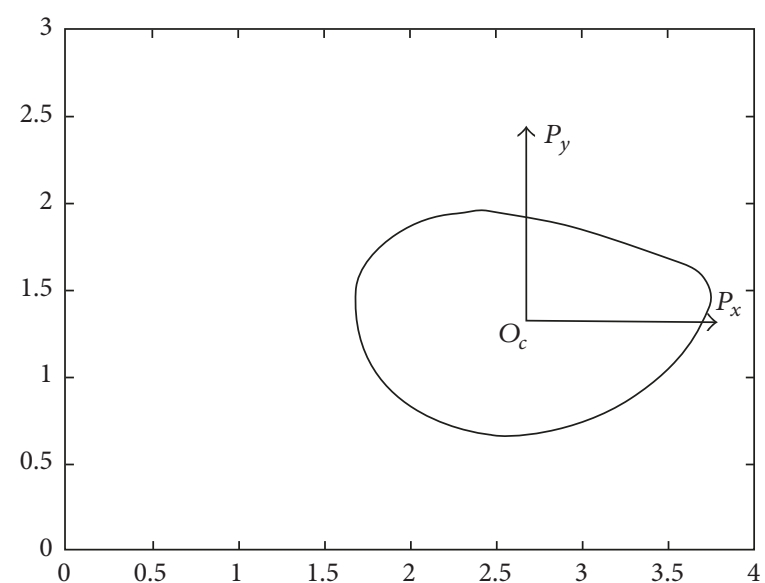

(b)

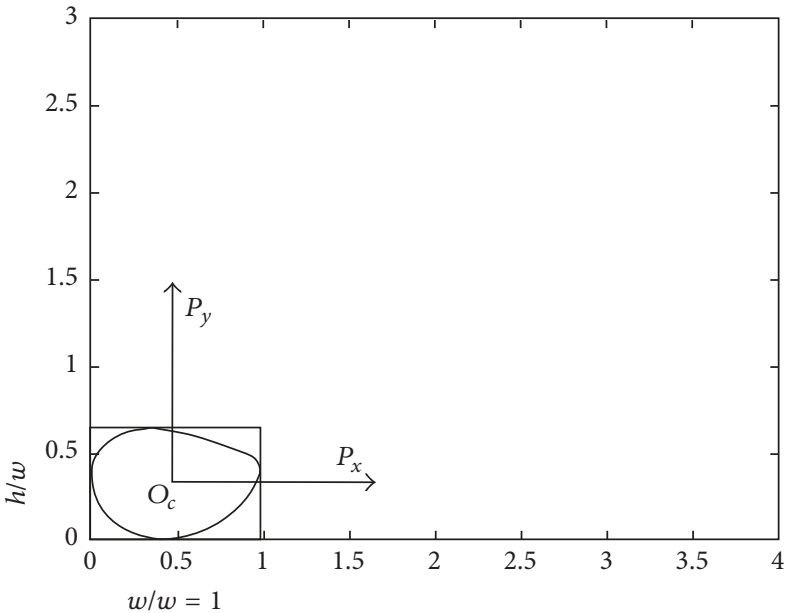

(d)

FIGURE 4: Curve normalization process. In (a), the original curve is shown; in (b), the curve is rotated around its center to align its major and minor principal axis of inertia of moment with $x$-axis and $y$-axis of fixed frame, respectively; in (c), a bounding box with width $w$ and height $h$ is produced to tightly confine the curve and $w / h$ is determined merely by the shape of the curve; in (d), we resize the curve with its bounding box so that width of bounding box becomes 1 and height $w / h$ and then relocate the bounding box to the origin of fixed frame.

5.2. Arc Length Parametrization. From a mathematical point of view, different parametrizations of the same curve correspond to different mathematical functions defined for the same curve. We have given two example functions in Section 2 that can define the unit circle: $z_{1}(t)=\cos (2 \pi t)+$ $j \sin (2 \pi t)$ and $z_{2}(t)=\cos \left[2 \pi\left(0.4 t^{3}+0.5 t^{2}+0.1 t\right)\right]+$ $j \sin \left[2 \pi\left(0.4 t^{3}+0.5 t^{2}+0.1 t\right)\right]$. Besides $z_{1}(t)$ and $z_{2}(t)$, there are a lot more functions to define the unit circle.

From a kinematic point of view, different parametrizations of the same curve correspond to different velocity patterns that a point uses to move along the same curve. For example, one parametrization tells the point to move quickly in the beginning and slowly in the end, while another parametrization may require the point to travel the same curve slowly in the beginning and quickly in the end. In these two situations, the point will spend different amount of time arriving at the same position on the path. Among numerous parametrizations, arc length parametrization corresponds to a point tracing the curve at a constant and unit speed; in this case, the time $t$ that the point uses to travel from the starting position to current position on the curve is numerically equal to the curve length or arc length between the starting and current position.

As explained in previous sections, FDs are different for different parametrizations. Suppose we have two geometrically identical paths with different parametrizations. By comparing their Fourier descriptors, these two paths would be deemed as different paths. To solve this problem, it is necessary to make the Fourier based comparison against the same parametrization. Hence, in order to match the normalized task and four-bar path generated from the curve normalization process, we need to obtain their Fourier descriptors under the arc length parametrization.

Consider a curve whose parametrization is given as a continuous function $z(t)=(x(t), y(t))$. Then, the arc length parametrization can be computed via the following steps: (a) by $s(t)=\int_{0}^{t}\left\|z^{\prime}(\tau)\right\| d \tau$, we get the arc length function $s$ against $t$; (b) compute $s^{-1}(t)$, the inverse function of $s(t)$, and we get 

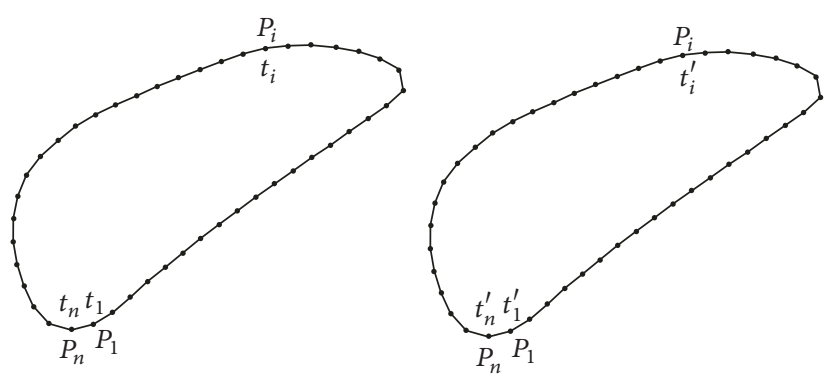

FIGURE 5: A sequence of points with two different parametrizations.

time $t$ function $t(s)=s^{-1}(t)$ against arc length; (c) substitute $t(s)$ into $z(t)$ and finally the arc length parametrization $z(t(s))=z_{s}(s)$ is obtained. However, it is impossible to derive an explicit formula for $s^{-1}(t)$.

In practice, the task path is usually given as a sequence of points $P_{i}(i=1, \ldots, n+1)$. Suppose two arbitrary parametrizations are associated with for these points: $P_{1}\left(t_{1}\right), P_{2}\left(t_{2}\right)$, $\ldots, P_{n}\left(t_{n}\right), P_{n+1}\left(t_{n+1}\right)$ and $P_{1}\left(t_{1}^{\prime}\right), P_{2}\left(t_{2}^{\prime}\right), \ldots, P_{n+1}\left(t_{n+1}^{\prime}\right)$. We specify $P_{1}$ as the starting position and $P_{n+1}$ as the end position and require that $P_{1}=P_{n+1}$. Since $P_{1}$ is the starting point, $t_{1}=$ $t_{1}^{\prime}=0$; in terms of $t_{i}$ and $t_{i}^{\prime}(i \neq 1)$, they indicate the time a point takes to travel from $P_{1}$ to $P_{i}$ under two parametrizations, respectively, which are usually different.

Now we seek to convert these two parametrizations to arc length parametrization by treating $P_{i}$ as vertices of a polygonal curve of $n$ sides, as shown in Figure 5. For both parametrizations, the arc length of $s_{i}$ from $P_{1}$ to $P_{i}$ can be calculated as

$$
s_{i}= \begin{cases}0, & i=1 \\ \sum_{k=1}^{i-1}\left|P_{k} P_{k+1}\right| & i=2, \ldots, n+1\end{cases}
$$

in which $\left|P_{k} P_{k+1}\right|$ denotes the length of line segment between $P_{k}$ and $P_{k+1}$. Using (26), both parametrizations are converted to the arc length parametrization $P_{1}\left(s_{1}\right), P_{2}\left(s_{2}\right)$, $\ldots, P_{n}\left(s_{n}\right), P_{n+1}\left(s_{n+1}\right)$. In order for the parameter to lie in the domain $[0,1]$, we divide $s_{i}$ by the total length of the polygonal curve $L$, where $L=s_{n+1}$. Hence, the arc length parametrization is finalized as $P_{1}\left(s_{1} / L\right)$, $P_{2}\left(s_{2} / L\right), \ldots, P_{n}\left(s_{n} / L\right), P_{n+1}\left(s_{n+1} / L\right)$.

By observing (26), the conversion from any parametrization to arc length parametrization is independent of the original parametrization, that is, the time sequence $t_{i}$ or $t_{i}^{\prime}$. Whatever parametrizations are given to the discrete path, the results of arc length parametrization are exactly the same. The geometry of the polygonal curve is the only factor that dictates the results of the arc length parametrization. Therefore, instead of coupling FDs with various parametrizations of the discrete path, we couple FDs directly with the underlying geometry of the discrete path by means of arc length parametrization.

5.3. Artificial Neural Network. The problem of matching the FDs of the normalized and arc length parametrized task

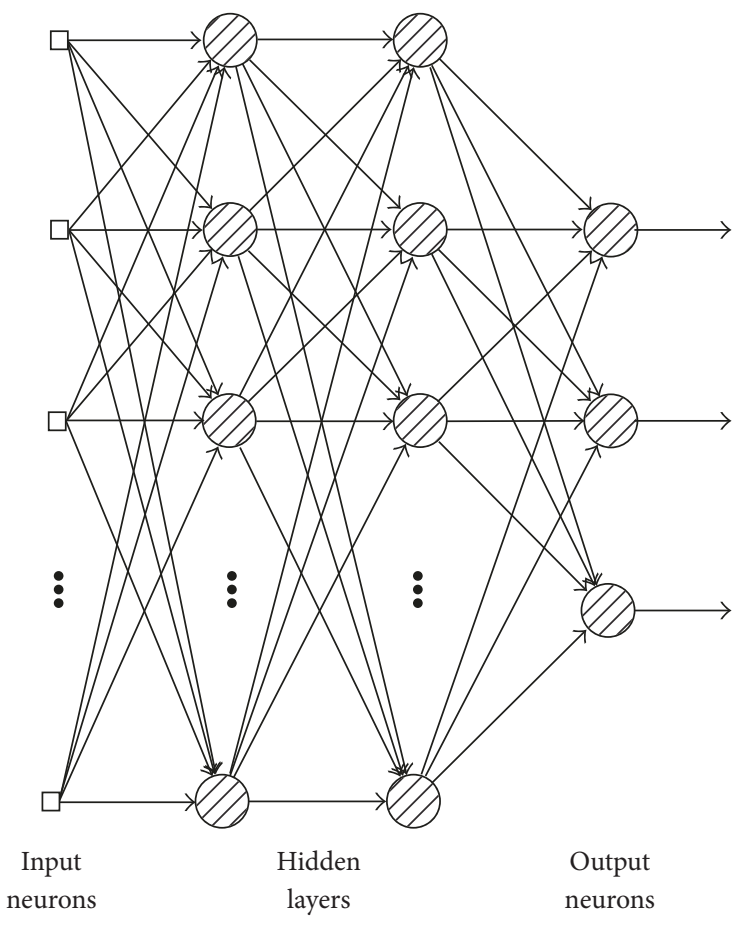

FIgURE 6: Typical neural network architecture.

and four-bar path is finding the optimal values of design parameters in (25). Instead of directly searching the five parameters, we use neural networks to establish the relationship between those parameters and the Fourier descriptors of the normalized and arc length parametrized four-bar path.

An artificial neural network is a computational structure consisting of a collection of interconnected elements, known as neurons, to estimate or approximate functions that are highly complex and nonlinear, and can have a large number of inputs and outputs (see Veelenturf [23] and Anastasio [24]). A typical neural network architecture is shown in Figure 6, which has one layer of input neurons, multiple layers of hidden neurons, and one layer of output neurons. In this paper, we use the widely adopted backpropagation (BP) algorithm to train a network to fit the relationship between $\left\{l_{21}, l_{31}, l_{41}, x_{c} / l_{3}, y_{c} / l_{3}\right\}$ and Fourier descriptors of the normalized and arc length parametrized four-bar path. $\mathrm{BP}$ is a supervised learning method which fits a function based on samples of input-output data pairs. For each input vector, the algorithm estimates the error between the actual and desired network outputs, and back propagates it from the output layer to hidden neurons to estimate the contribution of each hidden neuron to the output error. It calculates the gradient of each weight, which indicates the direction of error increase, and updates the weight in the opposite direction of the gradient.

The architecture of the proposed neural network has input neurons corresponding to FDs of the normalized and arc length parametrized four-bar path and output neurons corresponding to $\left\{l_{21}, l_{31}, l_{41}, x_{c} / l_{3}, y_{c} / l_{3}\right\}$. Our previous work [25] has shown that, for the four-bar coupler path, magnitudes of its high-order FDs are very close to zero; in other 
words, low-order descriptors are enough to represent the coupler path. Therefore, we take the FDs of order from -3 to 3 to be the input neurons of our neural network; that is, the number of input neurons is 14 , including the real and imaginary part of each descriptor; the number of output neurons is 5 , consistent with the number of design variables $\left\{l_{21}, l_{31}, l_{41}, x_{c} / l_{3}, y_{c} / l_{3}\right\}$.

In order to train the neural network, we use FDs of as many as 101,700 four-bar coupler curves, which are generated by the equal number of different combinations of the values of design variables $\left\{l_{21}, l_{31}, l_{41}, x_{c} / l_{3}, y_{c} / l_{3}\right\}$, and then processed by curve normalization and arc length parametrization; to see whether it can effectively approximate the relationship between the FDs and the design variables, we generate another 101, 700 testing cases to test the validity of our trained neural network.

In the training process, values of the five design variables are constrained to certain ranges. In practical applications, the ratio between any two links is not expected to be extremely large or small. So it is reasonable to predefine a max link ratio in the design process, say $K_{\max }$. Correspondingly, the minimum link ratio is $1 / K_{\max }$. Therefore, we vary three link ratios $l_{21}, l_{31}$, and $l_{41}$ in the range of $\left[1 / K_{\max }, K_{\max }\right]$. In this paper, $K_{\max }$ is taken to be 6 . Likewise, it is required that the coordinate of coupler point on coupler link needs to be dimensionally compatible with the length of coupler link. So we have similar constraints for $x_{c} / l_{3}$ and $y_{c} / l_{3}$ and $K_{\max }$ for them is 3 .

5.4. Restoring Method. Up to this point, we know the values of five design variables $\left\{l_{21}, l_{31}, l_{41}, x_{c} / l_{3}, y_{c} / l_{3}\right\}$. From the discussion of Section $4, \overline{\mathbf{P}}_{2}$ can be computed. According to (22), our goal is to find the four-bar curve $\mathbf{P}$ that best matches the task curve, say $\mathbf{T}$. Hence, we let $\mathbf{T}=\mathbf{P}$ and (22) becomes

$$
\mathbf{T}=\mathbf{P}_{1}+\mathbf{P}_{2} e^{j \theta_{1}}
$$

Next, there are three steps to implemented in order to match the size, orientation, and position of $\mathbf{T}$ and $\mathbf{P}$, during which $x_{0}, y_{0}, \theta_{1}, l_{2}$ could be found.

(1) Size Match between $\mathbf{T}$ and $\mathbf{P}$. Rotate $\mathbf{T}$ and $\overline{\mathbf{P}}_{2}$ to align their major principal axis with $x$-axis of fixed frame, respectively, and denote transformed curves to be $\mathscr{R}(\mathbf{T})$ and $\mathscr{R}\left(\overline{\mathbf{P}}_{2}\right)$. Compute the width or height of bounding box for $\mathscr{R}(\mathbf{T})$ and $\mathscr{R}\left(\overline{\mathbf{P}}_{2}\right)$ and denote the ratio as $w_{1} / w_{2}$ or $h_{1} / h_{2}$, which is the size ratio between $\mathbf{T}$ and $\overline{\mathbf{P}}_{2}$. According to (22), size of $\mathbf{P}$ is determined by $\mathbf{P}_{2}$ and $\mathbf{P}_{2}$ is equal to $l_{1} \overline{\mathbf{P}}_{2}$. Therefore, $l_{1}=w_{1} / w_{2}=h_{1} / h_{2}$.

(2) Orientation Match between $\mathbf{T}$ and $\mathbf{P}$. We obtain the value of $\mathbf{P}_{2}$ at Step (1) by $l_{1} \overline{\mathbf{P}}_{2}$. According to (22), the orientation difference between $\mathbf{P}$ and $\mathbf{P}_{2}$ lies in $\theta_{1}$. Therefore, $\theta_{1}$ is measured as the angle from the major principal axis of $\mathbf{P}_{2}$ to that of $\mathbf{T}$.

(3) Location Match between $\mathbf{T}$ and $\mathbf{P}$. Until now, we know $\mathbf{P}_{2} e^{j \theta_{1}}$. Then, we compute the centers for $\mathbf{T}$ and $\mathbf{P}_{2} e^{j \theta_{1}}$,

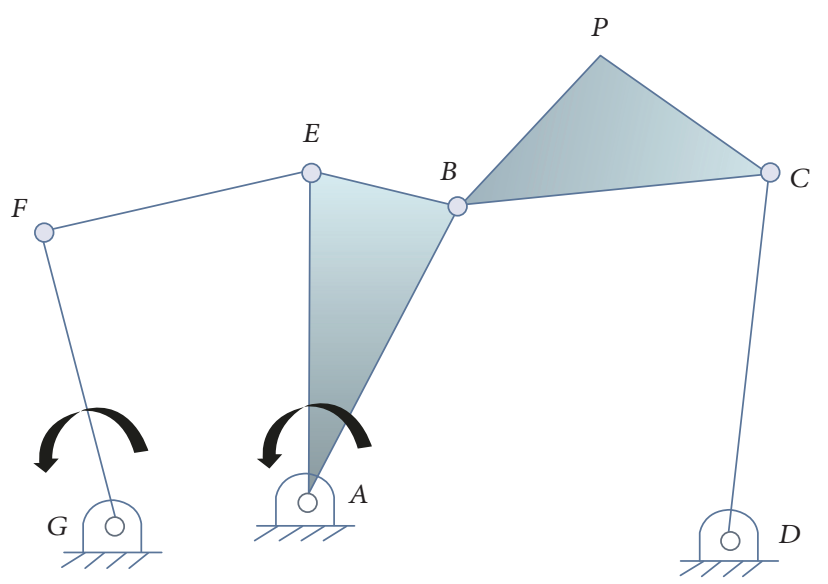

Figure 7: Watt II six-bar.

respectively, and denote them as $C_{1}$ and $C_{2}$. The distance vector from $C_{2}$ to $C_{1}$ is equal to $\mathbf{P}_{1}$.

Up to this point, the values of the nine design variables in (22) have been found except for $\phi_{0}$. As said earlier, $\phi_{0}$ only determines the starting point of curve and is irrelevant to position, orientation, size, and shape of curve. In practical applications, the starting point is chosen according to the user's need.

\section{Examples}

In this section, we present four examples to demonstrate the validity of the proposed parametrization-invariant FD based algorithm. In the first example, the task path is a coupler curve generated by a four-bar linkage of known dimensions, with three different parametrizations. For the second example, the task path is an arbitrarily given low harmonic curve, with two different parametrizations. For the third and final examples, the task path is a self-intersecting curve and an open curve, respectively, each with two parametrizations.

6.1. Generation of a Four-Bar Coupler Curve. In this example, we examine the effectiveness of our approach and make comparison with the traditional Fourier methods proposed by Chu et al. [15] and Wu et al. [20], respectively. The task path is generated by the coupler point $P$ of the Watt II six-bar shown in Figure 7. Four-bar $A E F G$ functioning as driving linkage is serially chained with four-bar $A B C D$, of which $P$ serves as the coupler point to trace out the coupler curve. AEFG must be a double-crank mechanism in order for $G F$ and $A E$ to rotate in full circle, respectively. When $G F$ rotates with a constant angular speed, $A E$ will rotate with a varying speed. Hence, three different parametrizations are produced by altering the lengths of $G F, F E$, and $A G$ to change the rotating function of $A E$. Links $A B, B C, C D, B P$, and $C P$ remain the same to keep the shape of the closed curve traced by $P$ under the three parametrizations. Nine design parameters are given as $x_{0}=2.2, y_{0}=3.5, l_{1}=4.4, l_{21}=0.5$, $l_{31}=2.8, l_{41}=2.7, x_{c} / l_{3}=1.0, y_{c} / l_{3}=0.8, \theta_{1}=0.55$. Three sets of $A E, E F, F G$, and $A G$ are given as follows: 


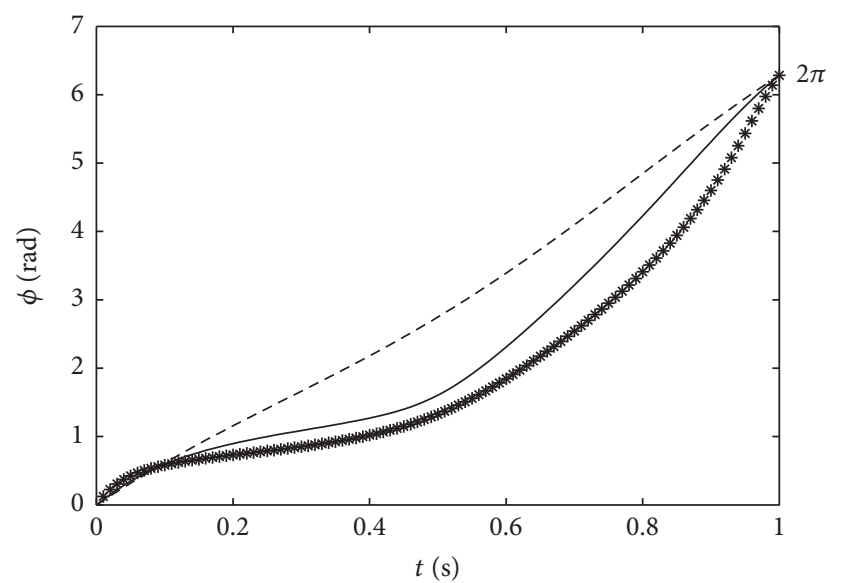

- Parametrization 1

* Parametrization 2

- - Parametrization 3

FIGURE 8: Rotation functions of link $A B$ for three parametrizations.

TABLE 1: Magnitudes of FDs of the task curve for three different parametrizations.

\begin{tabular}{lccc}
\hline & I & II & III \\
\hline $\mathrm{FD}_{-3}$ & 0.9743 & 1.0661 & 0.6969 \\
$\mathrm{FD}_{-2}$ & 1.7290 & 1.5949 & 1.4765 \\
$\mathrm{FD}_{-1}$ & 2.3956 & 2.0666 & 3.2828 \\
$\mathrm{FD}_{0}$ & 16.7382 & 17.2055 & 17.0292 \\
$\mathrm{FD}_{1}$ & 1.7154 & 1.4730 & 2.3377 \\
$\mathrm{FD}_{2}$ & 1.1184 & 1.0410 & 0.7418 \\
$\mathrm{FD}_{3}$ & 0.4841 & 0.5770 & 0.2318 \\
\hline
\end{tabular}

$$
\begin{array}{ccc}
\text { Parametrization I: } A E=0.50 & E F=1.60 \\
F G=1.60 & A G=0.35, \\
\text { Parametrization } & \text { II: } A E=0.50 & E F=0.80 \\
F G=0.75 & A G=0.40, \\
\text { Parametrization III: } A E=0.50 & E F=2.35 \\
F G=2.40 & A G=0.10 .
\end{array}
$$

Rotation functions of link $A B$ corresponding to each parametrization are plotted in Figure 8. By observing the figure, we can see that parametrization III is the perfect parametrization in which link $A B$ rotates uniformly while parametrization I and parametrization II deviate from the perfect parametrization, which indicates that link $A B$ rotates with a varying angular velocity.

For these different parametrizations, their corresponding sets of FDs are listed in Table 1. It is clear from the table that different parametrizations yield different FDs, even though they define the same curve. After arc length parametrization, we obtain three new sets of Fourier in Table 2.

By observing the data in Table 2, three sets of FDs are almost the same just as we discussed in previous sections. In order to demonstrate the effectiveness of our approach,
TABLE 2: Magnitudes of FDs of the task curve for three different parametrizations after the arc-length reparametrization.

\begin{tabular}{lccc}
\hline & $\mathrm{I}$ & $\mathrm{II}$ & $\mathrm{III}$ \\
\hline $\mathrm{FD}_{-3}$ & 0.4127 & 0.4155 & 0.4106 \\
$\mathrm{FD}_{-2}$ & 0.1928 & 0.1931 & 0.1911 \\
$\mathrm{FD}_{-1}$ & 3.7141 & 3.7148 & 3.7145 \\
$\mathrm{FD}_{-0}$ & 17.8644 & 17.8649 & 17.8641 \\
$\mathrm{FD}_{1}$ & 1.9238 & 1.9240 & 1.9242 \\
$\mathrm{FD}_{2}$ & 0.2511 & 0.2522 & 0.2520 \\
$\mathrm{FD}_{3}$ & 0.1528 & 0.1522 & 0.1520 \\
\hline
\end{tabular}

TABLE 3: The design parameters of synthesized four-bar linkages for three parametrizations by our method. All angles are in rad.

\begin{tabular}{lccc}
\hline Parametrization & I & II & III \\
\hline$l_{21}$ & 0.5045 & 0.5072 & 0.5077 \\
$l_{31}$ & 2.8020 & 2.8039 & 2.7963 \\
$l_{41}$ & 2.7070 & 2.7096 & 2.7080 \\
$x_{c} / l_{3}$ & 1.0028 & 0.9942 & 1.0011 \\
$y_{c} / l_{3}$ & 0.8075 & 0.7908 & 0.8036 \\
$l_{1}$ & 4.3286 & 4.3549 & 4.3228 \\
$\theta_{1}$ & 0.5495 & 0.5615 & 0.5599 \\
$x_{0}$ & 2.3715 & 2.4686 & 2.4719 \\
$y_{0}$ & 3.5416 & 3.4464 & 3.5058 \\
\hline
\end{tabular}

TABLE 4: Magnitudes of FDs of three synthesized coupler curves for three parametrizations after the arc-length reparametrization by our method.

\begin{tabular}{lccc}
\hline & I & II & III \\
\hline $\mathrm{FD}_{-3}$ & 0.4118 & 0.4118 & 0.4120 \\
$\mathrm{FD}_{-2}$ & 0.1898 & 0.1918 & 0.1906 \\
$\mathrm{FD}_{-1}$ & 3.7119 & 3.7090 & 3.7159 \\
$\mathrm{FD}_{0}$ & 17.8644 & 17.8649 & 17.8641 \\
$\mathrm{FD}_{1}$ & 1.9250 & 1.9216 & 1.9246 \\
$\mathrm{FD}_{2}$ & 0.2560 & 0.2585 & 0.2573 \\
$\mathrm{FD}_{3}$ & 0.1550 & 0.1539 & 0.1547 \\
\hline
\end{tabular}

we compare the synthesis results with those generated by the traditional synthesis approaches (Chu et al. [15]; Wu et al. [20]), respectively.

First, the results of our method are presented. In Table 3, the resulting design parameters corresponding to three parametrizations are displayed. We can see that those parameters are close to those of the four-bar mechanism presented at the beginning that generates the task curve. In Table 4, FDs for three parametrizations are shown. Compared with each corresponding parametrization in Table 2, the synthesized curves match perfectly the task curve.

Second, we use the approach presented by $\mathrm{Wu}$ et al. [20] to obtain three four-bar mechanisms and show results in Tables 5 and 6. According to Table 5, the values of design parameters are quite different from the task fourbar mechanism. Also from Tables 6 and 2, difference in FDs is observable. The graphical comparisons are shown in 
TABLE 5: The design parameters of synthesized four-bar linkages under three parametrizations by Wu's method. All angles are in rad.

\begin{tabular}{lccc}
\hline Parametrization & I & II & III \\
\hline$l_{21}$ & 0.7980 & 0.8554 & 0.6052 \\
$l_{31}$ & 1.7515 & 4.9052 & 1.8951 \\
$l_{41}$ & 1.9051 & 4.9980 & 1.8050 \\
$x_{c} / l_{3}$ & 0.8903 & 0.2190 & 1.3764 \\
$y_{c} / l_{3}$ & 0.6551 & 0.4433 & 0.6998 \\
$l_{1}$ & 4.2372 & 3.2539 & 4.3040 \\
$\theta_{1}$ & 1.0531 & 2.7821 & 0.7563 \\
$x_{0}$ & 10.4813 & 10.8015 & 5.4999 \\
$y_{0}$ & 0.7741 & 3.4920 & 2.1181 \\
\hline
\end{tabular}

TABLE 6: Magnitudes of FDs of three synthesized coupler curves by Wu's method.

\begin{tabular}{lccc}
\hline & $\mathrm{I}$ & $\mathrm{II}$ & $\mathrm{III}$ \\
\hline $\mathrm{FD}_{-3}$ & 0.3588 & 0.3148 & 0.3954 \\
$\mathrm{FD}_{-2}$ & 0.2429 & 0.2303 & 0.2058 \\
$\mathrm{FD}_{-1}$ & 3.8635 & 3.8858 & 3.7170 \\
$\mathrm{FD}_{0}$ & 18.1680 & 18.6918 & 17.8926 \\
$\mathrm{FD}_{1}$ & 1.5478 & 1.4226 & 1.8367 \\
$\mathrm{FD}_{2}$ & 0.2866 & 0.2687 & 0.2839 \\
$\mathrm{FD}_{3}$ & 0.1569 & 0.1279 & 0.1532 \\
\hline
\end{tabular}

Figures 9, 10, and 11, including the synthesized four-bar mechanism by our approach. For the three parametrizations, III is the perfect parametrization as indicated earlier and therefore Wu's method can yield a good match with original curve. The other two parametrizations twist perfect parametrization to the extent that reduces the exactness of Wu's method.

Then, we use the approach proposed by Chu et al. [15] to synthesize three four-bar mechanisms and show results in Tables 7 and 8. The graphical comparisons are revealed in Figures 9, 10, and 11. By comparing results generated by Chu's method with those by Wu's method, we find that both approaches can output good match when the parametrization is perfect, as in the case of parametrization III. However, the more the parametrization differs from the perfect one, the more their synthesized curves deviate from the task curve, which is justified by comparing synthesized curves of both methods under parametrization I with those of II in Figures 9 and 10. Clearly, the results for I are better than those of II because I is closer to perfect parametrization than II, as clearly shown in Figure 8.

6.2. Generation of an Arbitrary Closed Curve. Now consider the problem of synthesizing a four-bar mechanism for generating an arbitrarily specified path. The task path is given as

$$
\begin{aligned}
z(f(t))= & (100+20 j)+(20+14 j) e^{-j 2 \pi f(t)} \\
& +(45-30 j) e^{j 2 \pi f(t)}+(6+4 j) e^{-j 4 \pi f(t)} \\
& +(9-3 j) e^{j 4 \pi f(t)},
\end{aligned}
$$

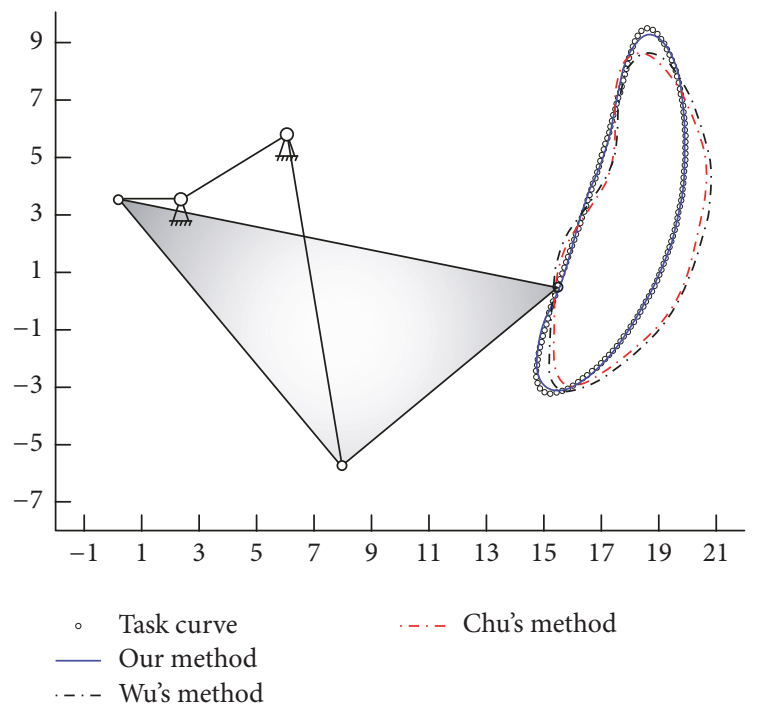

FIGURE 9: Four-bar coupler curve generation for parametrization I. The task curve, synthesized curves by our method, Wu's method, and Chu's method are represented by circular dots, blue solid curve, black dashed curve, and red dashed curve, respectively.

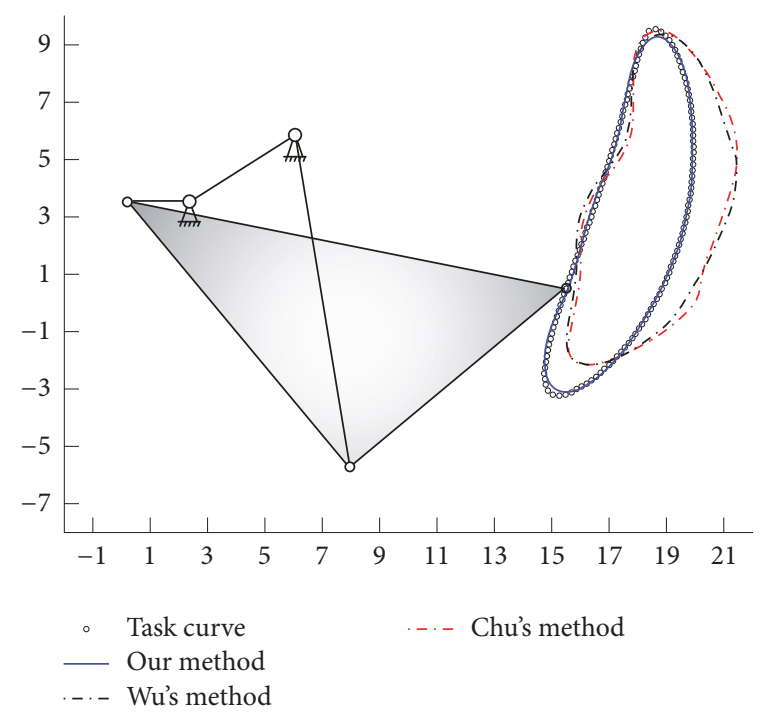

FIGURE 10: Four-bar coupler curve generation for parametrization II. The task curve, synthesized curves by our method, Wu's method, and Chu's method are represented by circular dots, blue solid curve, black dashed curve, and red dashed curve, respectively.

where $f(t)=t(0 \leq t \leq 1)$ for the first parametrization and $f(t)=0.5 t^{2}+0.5 t(0 \leq t \leq 1)$ for the second parametrization.

In this example, we compare the results of approach against those by Wu's method. The graphical comparisons of the synthesis results for the first and second parametrizations are shown in Figures 12 and 13, respectively, together with the synthesized four-bar mechanisms by our approach. In both cases, the synthesized four-bar curves of our approach are independent of parametrization and therefore approximate the task path in a consistent manner. In contrast, Wu's method outputs different curves for the two parametrizations, and the 


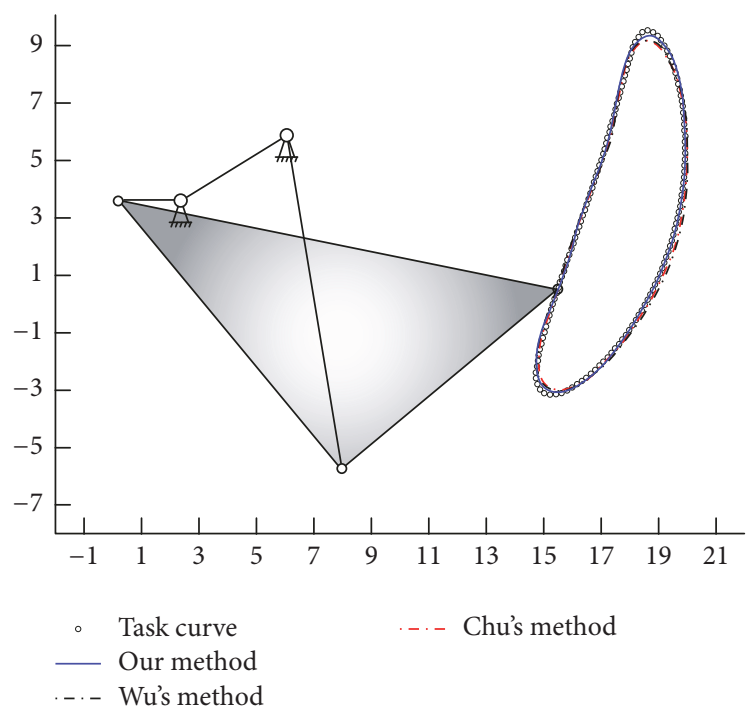

FIGURE 11: Four-bar coupler curve generation for parametrization III. The task curve, synthesized curves by our method, Wu's method, and Chu's method are represented by circular dots, blue solid curve, black dashed curve, and red dashed curve, respectively.

TABLE 7: The design parameters of synthesized four-bar linkages under three parametrizations by Chu's method. All angles are in rad.

\begin{tabular}{lccc}
\hline Parametrization & I & II & III \\
\hline$l_{21}$ & 0.8051 & 0.9011 & 0.6050 \\
$l_{31}$ & 1.8950 & 4.9550 & 1.7516 \\
$l_{41}$ & 2.0522 & 5.0032 & 1.8452 \\
$x_{c} / l_{3}$ & 0.7978 & 0.2112 & 1.2720 \\
$y_{c} / l_{3}$ & 0.6179 & 0.4468 & 0.5649 \\
$l_{1}$ & 4.1185 & 3.0472 & 4.8738 \\
$\theta_{1}$ & 3.3154 & 0.3687 & 1.0926 \\
$x_{0}$ & 10.8081 & 11.3207 & 6.3888 \\
$y_{0}$ & 0.6750 & 3.5521 & 1.1477 \\
\hline
\end{tabular}

TABLE 8: Magnitudes of FDs of three synthesized coupler curves by Chu's method.

\begin{tabular}{lccc}
\hline & I & II & III \\
\hline $\mathrm{FD}_{-3}$ & 0.3704 & 0.3994 & 0.3939 \\
$\mathrm{FD}_{-2}$ & 0.2667 & 0.2338 & 0.2020 \\
$\mathrm{FD}_{-1}$ & 3.7364 & 3.8833 & 3.6882 \\
$\mathrm{FD}_{0}$ & 18.1640 & 18.7623 & 17.8883 \\
$\mathrm{FD}_{1}$ & 1.5323 & 1.4473 & 1.8343 \\
$\mathrm{FD}_{2}$ & 0.3102 & 0.3250 & 0.2736 \\
$\mathrm{FD}_{3}$ & 0.1415 & 0.0523 & 0.1512 \\
\hline
\end{tabular}

generated curve for the second parametrization (when $f(t)$ is quadratic) is less accurate compared to the one for the first parametrization.

6.3. Generation of a Self-Intersecting Curve. Consider the task of generating a self-intersecting curve, whose function is given as

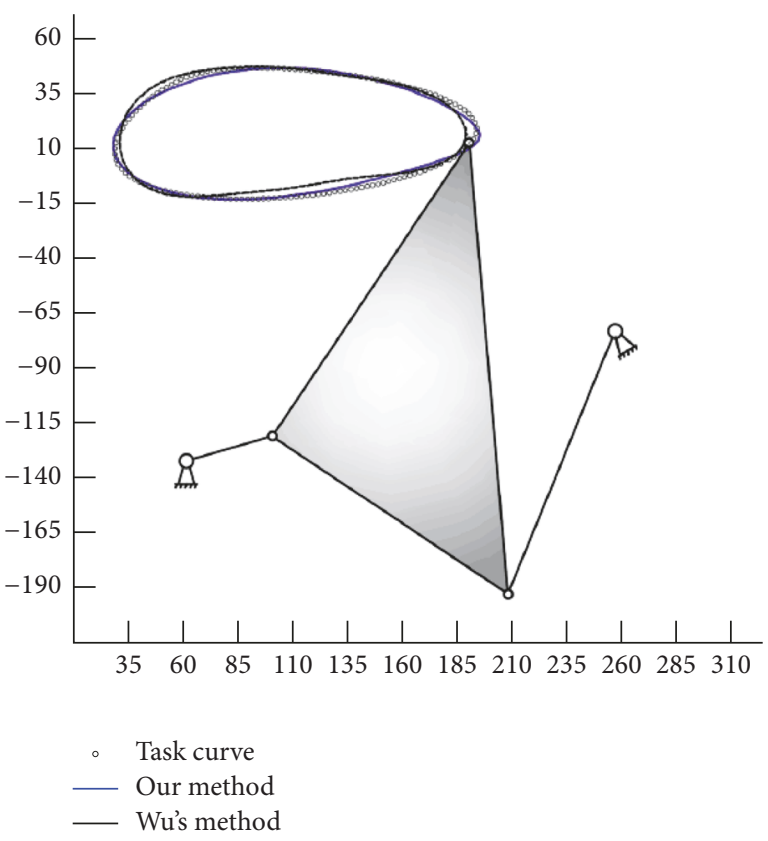

FIGURE 12: Arbitrary curve generation for the first parametrization. The task curve, synthesized curves by our method, and Wu's method are represented by circular dots, blue solid curve, and black solid curve, respectively.

$$
\begin{aligned}
z(f(t))= & (41.75+192.35 j) \\
& +(-21.76+42.07 j) e^{-j 2 \pi f(t)} \\
& +(53.69+6.67 j) e^{j 2 \pi f(t)} \\
& +(-11.36+13.66 j) e^{-j 4 \pi f(t)} \\
& +(-0.56-8.44 j) e^{j 4 \pi f(t)} \\
& +(-5.80+5.06 j) e^{-j 6 \pi f(t)} \\
& +(-0.15-2.13 j) e^{j 6 \pi f(t)},
\end{aligned}
$$

where $f(t)=t(0 \leq t \leq 1)$ for the first parametrization and $f(t)=0.5 t^{2}+0.5 t(0 \leq t \leq 1)$ for the second parametrization.

We still compare the results of our approach against those by Wu's method. The graphical comparisons of the synthesis results for the first and second parametrizations are shown in Figures 14 and 15, respectively, together with the synthesized four-bar mechanisms by our approach. In both cases, the synthesized four-bar curves of our approach are independent of parametrization and approximate the task path in a consistent and accurate manner. In contrast, Wu's method outputs different curves for the two parametrizations, and the generated curve for the second parametrization (when $f(t)$ is quadratic) is less accurate compared to the one for the first parametrization.

6.4. Generation of an Open Curve. Consider the task of generating an open curve, whose function is given as 


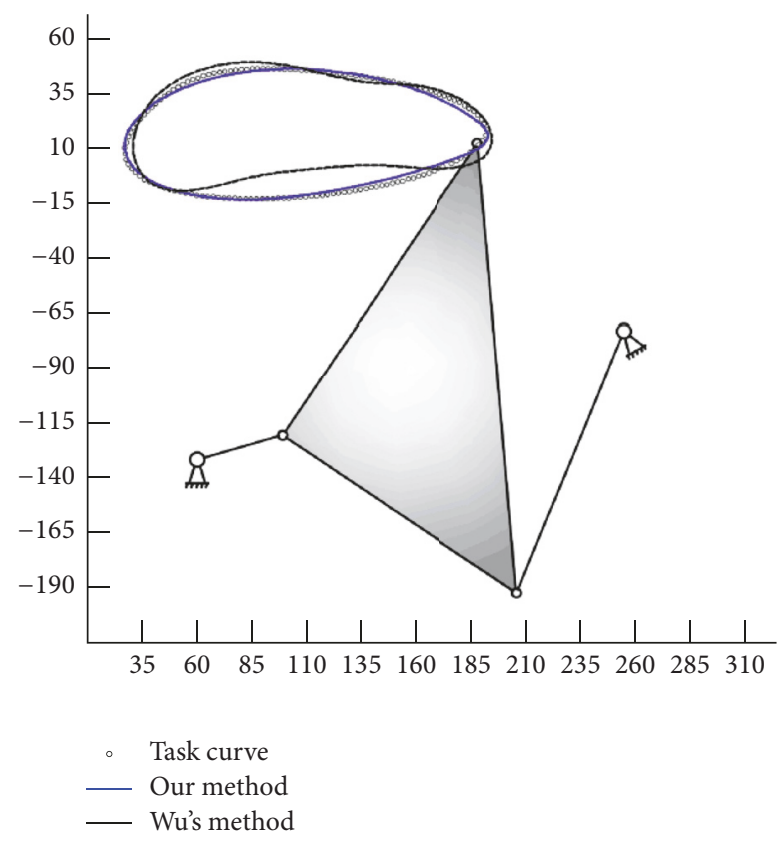

FIGURE 13: Arbitrary curve generation for the second parametrization. The task curve, synthesized curves by our method, and Wu's method are represented by circular dots, blue solid curve, and black solid curve, respectively.

$$
\begin{aligned}
z(f(t))= & (26.36+21.48 j)+(3.33+1.74 j) e^{-j 2 \pi f(t)} \\
& +(4.21+8.64 j) e^{j 2 \pi f(t)} \\
& +(0.89-0.15 j) e^{-j 4 \pi f(t)} \\
& +(0.14-0.66 j) e^{j 4 \pi f(t)} \\
& +(0.17-0.13 j) e^{-j 6 \pi f(t)} \\
& +(0.07-0.06 j) e^{j 6 \pi f(t)}
\end{aligned}
$$

where $f(t)=t(0.2 \leq t \leq 0.6)$ for the first parametrization and $f(t)=0.83 t^{2}+0.33 t+0.10(0.2 \leq t \leq 0.6)$ for the second parametrization.

We again compare the results of our approach against those by Wu's method. The graphical comparisons of the synthesis results for the first and second parametrizations are shown in Figures 16 and 17, respectively, together with the synthesized four-bar mechanisms by our approach. In both cases, the synthesized four-bar curves of our approach are independent of parametrization and approximate the task path in a consistent and accurate manner. In contrast, Wu's method outputs different curves for the two parametrizations, and the generated curve for the second parametrization (when $f(t)$ is quadratic) is less accurate compared to the one for the first parametrization.

\section{Conclusions}

In this paper, we apply the technique of arc length parametrization to avoid the influence of parametrization on

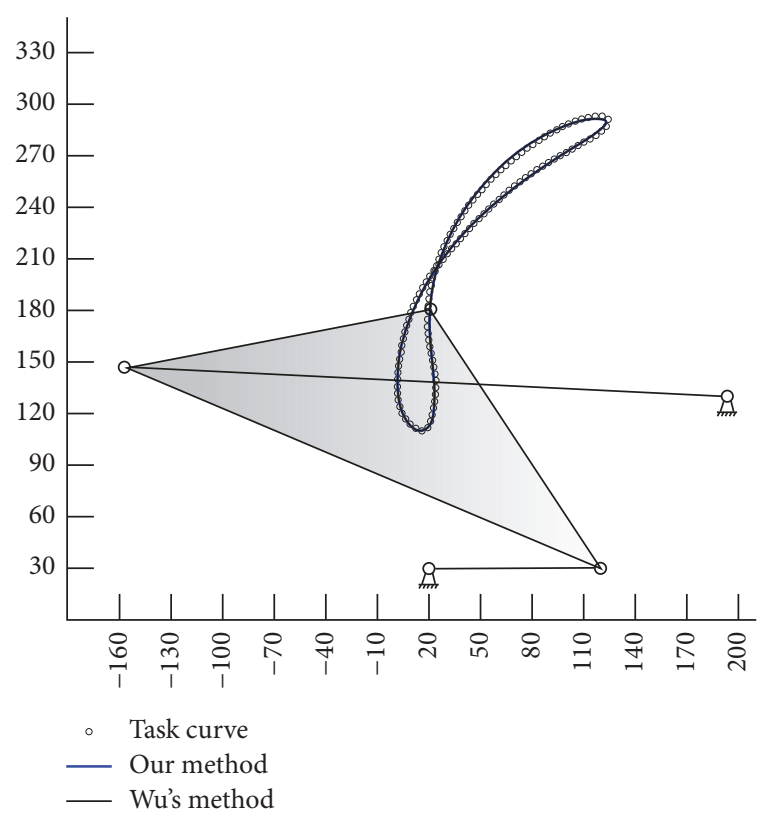

FIGURE 14: Self-intersecting curve generation for the first parametrization. The task curve, synthesized curves by our method, and Wu's method are represented by circular dots, blue solid curve, and black solid curve, respectively.

FD based approach to four-bar path synthesis. We manage to decouple the design space of the four-bar mechanism through matching the shape, size, orientation, and location of the task path, respectively. Furthermore, with the aid of backpropagation artificial neural network, five design parameters can be efficiently determined once the network is trained; the rest of four design parameters are then separately found by the proposed restoring method that implements the step-by-step matching of size, orientation, and location with the task curve. Finally, the synthesis results for four-bar curve and arbitrary curve generation are compared with the traditional Fourier based methods to show that our method can trace the task curve in a consistent manner, invariant to the parametrization of the task curve.

\section{Appendix}

The curve is assumed to be a simple closed polygon of $n$ sides where $\left(x_{i}, y_{i}\right)$ represents the $i$ th vertex of the polygon and $\left(x_{0}, y_{0}\right)$ is considered identical to $\left(x_{n}, y_{n}\right)$. All the calculations are done with respect to the length elements of the polygon.

Step 1. Evaluate the length $L$ of the curve. For that, let $l_{i}$ denote the $i$ th edge of the polygon. Then,

$$
\begin{aligned}
& l_{i}=\sqrt{\left(x_{i-1}-x_{i}\right)^{2}+\left(y_{i-1}-y_{i}\right)^{2}}, \\
& L=\sum_{i=1}^{n} l_{i} .
\end{aligned}
$$




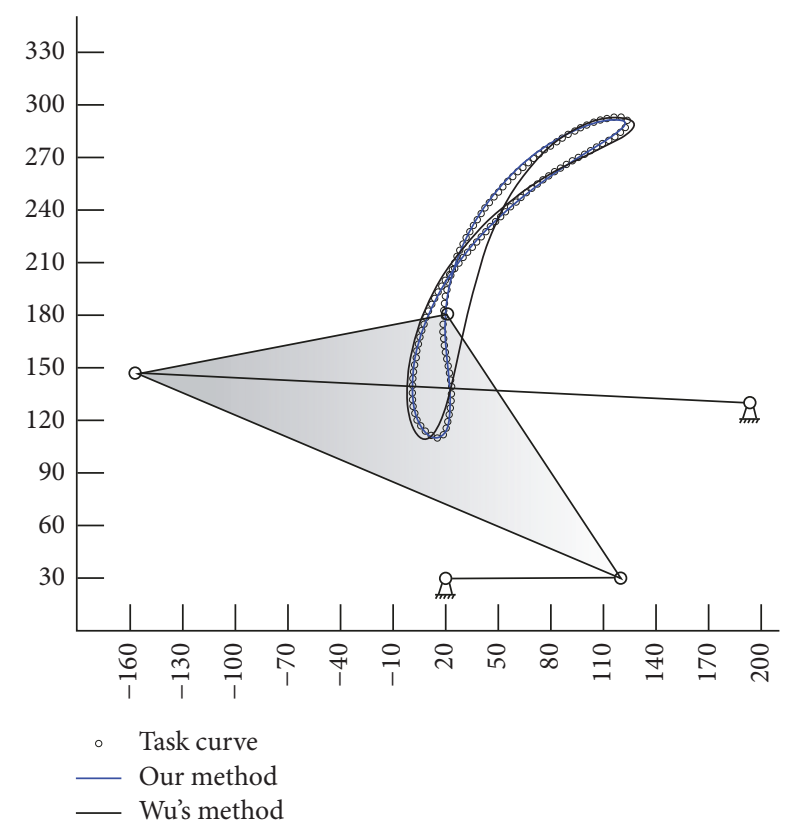

FIGURE 15: Self-intersecting curve generation for the second parametrization. The task curve, synthesized curves by our method, and Wu's method are represented by circular dots, blue solid curve, and black solid curve, respectively.

Step 2. Determine the location of the center of gravity (c.g.) of the curve given by $\left(c_{x}, c_{y}\right)$ :

$$
\begin{aligned}
& c_{x}=\frac{1}{2 L}\left(\sum_{i=1}^{n}\left(x_{i-1}+x_{i}\right) \sqrt{\left(x_{i-1}-x_{i}\right)^{2}+\left(y_{i-1}-y_{i}\right)^{2}}\right), \\
& c_{y}=\frac{1}{2 L}\left(\sum_{i=1}^{n}\left(y_{i-1}+y_{i}\right) \sqrt{\left(x_{i-1}-x_{i}\right)^{2}+\left(y_{i-1}-y_{i}\right)^{2}}\right) .
\end{aligned}
$$

Step 3. Evaluate the moments of inertia $I_{x x}, I_{y y}$, and $I_{x y}$ of the polygon with respect to its c.g. $\left(c_{x}, c_{y}\right)$ :

$$
\begin{aligned}
I_{x x} & =\frac{1}{3} \sum_{i=1}^{n}\left\{l _ { i } \left[\left(y_{i-1}-c_{y}\right)^{2}+\left(y_{i}-c_{y}\right)^{2}\right.\right. \\
& \left.\left.+\left(y_{i-1}-c_{y}\right)\left(y_{i}-c_{y}\right)\right]\right\}, \\
I_{y y} & =\frac{1}{3} \sum_{i=1}^{n}\left\{l _ { i } \left[\left(x_{i-1}-c_{x}\right)^{2}+\left(x_{i}-c_{x}\right)^{2}\right.\right. \\
& \left.\left.+\left(x_{i-1}-c_{x}\right)\left(x_{i}-c_{x}\right)\right]\right\}, \\
I_{x y} & =\frac{1}{6} \sum_{i=1}^{n}\left\{l _ { i } \left[\left(x_{i-1}-c_{x}\right)\left(y_{i}-c_{y}\right)\right.\right. \\
& \left.+\left(x_{i}-c_{x}\right)\left(y_{i-1}-c_{y}\right)\right]
\end{aligned}
$$

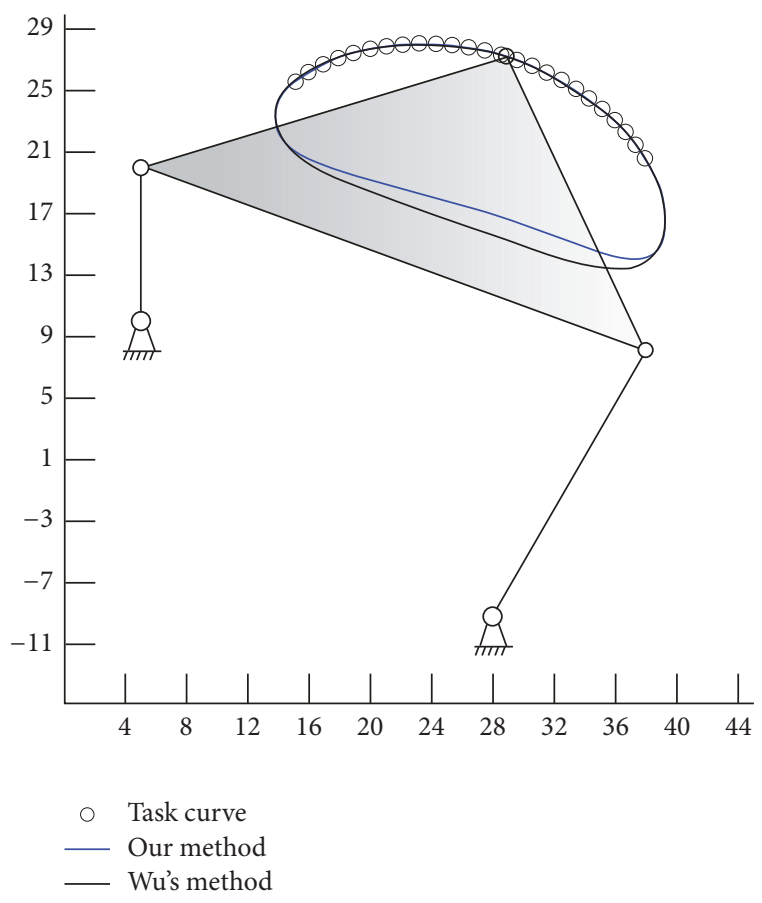

FIGURE 16: Open curve generation for the first parametrization. The task curve, synthesized curves by our method, and Wu's method are represented by circular dots, blue solid curve, and black solid curve, respectively.

$$
\begin{aligned}
& +2 l_{i}\left[\left(x_{i-1}-c_{x}\right)\left(y_{i-1}-c_{y}\right)\right. \\
& \left.\left.+\left(x_{i}-c_{x}\right)\left(y_{i}-c_{y}\right)\right]\right\} .
\end{aligned}
$$

Step 4. Determine the direction, $\alpha$, of the major principal axis with respect to the $x$-axis:

$$
\alpha=\frac{1}{2} \arctan \left(\frac{2 I_{x y}}{I_{y y}-I_{x x}}\right) .
$$

The direction of the major principal axis with respect to the $x$-axis is given by $\alpha$ if $I_{x x}<I_{y y}$ or $\alpha+\pi / 2$ if $I_{x x}>I_{y y}$.

Step 5. Rotate the polygon by an angle- $\alpha$ by the rotation to the vertices of the polygon in order to align the major principal axis with $x$-axis:

$$
\left(\begin{array}{l}
x_{i} \\
y_{i}
\end{array}\right)_{\text {new }}=\left[\begin{array}{cc}
\cos \alpha & \sin \alpha \\
-\sin \alpha & \cos \alpha
\end{array}\right]\left(\begin{array}{l}
x_{i} \\
y_{i}
\end{array}\right)_{\text {old }} .
$$

Step 6. Evaluate the width $w$ of the bounding box of the resulting polygon. Let $\left(P_{x}, P_{y}\right)$ and $\left(Q_{x}, Q_{y}\right)$ represent the corners of the bounding box obtained by comparing the coordinates of the points of the polygon. Then the width $w$ and height $h$ of the bounding box are $\left|Q_{x}-P_{x}\right|$ and $\left|Q_{y}-P_{y}\right|$, respectively. Since the major principal axis is aligned with the $x$-axis after rotation, $w>h$. 


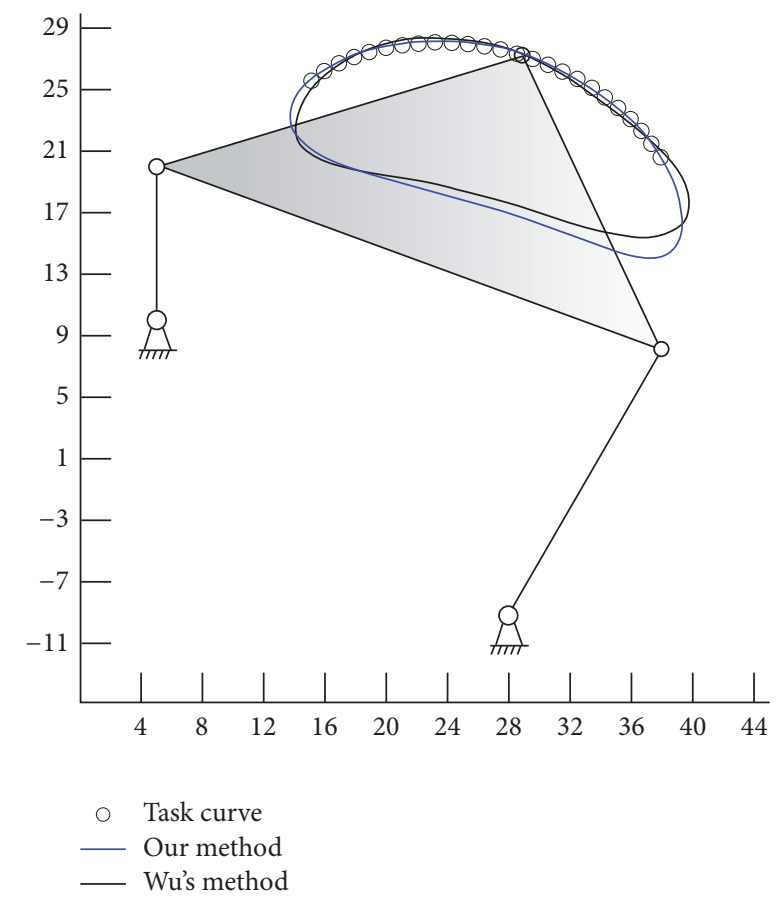

FIGURE 17: Open curve generation for the second parametrization. The task curve, synthesized curves by our method, and Wu's method are represented by circular dots, blue solid curve, and black solid curve, respectively.

Step 7. Bring the polygon to its normalized configuration:

$$
\begin{aligned}
& \left(\begin{array}{c}
x_{i} \\
y_{i} \\
1
\end{array}\right)_{\text {normalized }} \\
& =\left[\begin{array}{ccc}
\frac{\cos \alpha}{w} & \frac{\sin \alpha}{w} & -\frac{P_{x}}{w} \\
-\frac{\sin \alpha}{w} & \frac{\cos \alpha}{w} & -\frac{P_{y}}{w} \\
0 & 0 & 1
\end{array}\right]\left(\begin{array}{c}
x_{i} \\
y_{i} \\
1
\end{array}\right)_{\text {old }} .
\end{aligned}
$$

Step 8. Normalize the direction of the curve. To compute the signed area of the resulting polygon, the following shoelace formula (see Beyer [26]) is used:

$$
A=\frac{1}{2}\left|\sum_{i=1}^{n-1} x_{i} y_{i+1}-\sum_{i=1}^{n-1} x_{i+1} y_{i}+x_{n} y_{1}-x_{1} y_{n}\right| \text {, }
$$

where $A$ is the area of the polygon. If $A>0$, the vertices of the polygon are arranged in a counterclockwise order; if $A<0$, the vertices of the polygon are arranged in a clockwise order. We require that the direction of the normalized polygon is counterclockwise. In the case of $A<0$, the order of the vertices will be reversed.

\section{Disclosure}

All findings and results presented in this paper are those of the authors and do not represent those of the funding agencies.

\section{Conflicts of Interest}

The authors declare that there are no conflicts of interest regarding the publication of this paper.

\section{Acknowledgments}

The work has been financially supported by the Fundamental Research Funds for the Central Universities of China (no. 2682017CX037 and no. 2682017CX036) and the National Natural Science Foundation of China (no. 51305368).

\section{References}

[1] L. F. Costa and R. M. Cesar, Shape Analysis and Classification: Theory and Practice, CRC Press, Boca Raton, FL, USA, 2001.

[2] F. Freudenstein, "Harmonic analysis of crank-and-rocker mechanisms with application," Journal of Applied Mechanics, vol. 26, no. 4, pp. 673-675, 1959.

[3] H. Funabashi and F. Freudenstein, "Performance criteria for high-speed crank-and-rocker linkages - 1. plane crank-androcker linkages," Journal of Mechanical Design, vol. 101, no. 1, pp. 20-25, 1979.

[4] K. Farhang, A. Midha, and A. K. Bajaj, "Synthesis of harmonic motion generating linkages - part I: function generation," Journal of Mechanisms, Transmissions, and Automation in Design, vol. 110, no. 1, pp. 16-21, 1988.

[5] K. Farhang, A. Midha, and A. K. Bajaj, "Synthesis of harmonic motion generating linkages - part II: path and motion generation," Journal of Mechanisms, Transmissions, and Automation in Design, vol. 110, no. 1, pp. 22-27, 1988.

[6] J. Chu and W. Cao, "Synthesis of coupler curves of planar fourbar linkages through fast fourier transform," Chinese Journal of Mechanical Engineering, vol. 29, no. 5, pp. 117-122, 1993.

[7] J. McGarva and G. Mullineux, "Harmonic representation of closed curves," Applied Mathematical Modelling, vol. 17, no. 4, pp. 213-218, 1993.

[8] J. R. McGarva, "Rapid search and selection of path generating mechanisms from a library," Mechanism and Machine Theory, vol. 29, no. 2, pp. 223-235, 1994.

[9] I. Ullah and S. Kota, "Optimal synthesis of mechanisms for path generation using fourier descriptors and global search methods," Journal of Mechanical Design, vol. 119, no. 10, pp. 504510, 1997.

[10] X. Nie and V. Krovi, "Fourier methods for kinematic synthesis of coupled serial chain mechanisms," Journal of Mechanical Design, vol. 127, no. 2, pp. 232-241, 2005.

[11] J. Chu and J. Sun, "A new approach to dimension synthesis of spatial four-bar linkage through numerical atlas method," Journal of Mechanisms and Robotics, vol. 2, no. 4, Article ID 041004, 2010.

[12] J. Chu and J. Sun, "Numerical atlas method for path generation of spherical four-bar mechanism," Mechanism and Machine Theory, vol. 45, no. 6, pp. 867-879, 2010.

[13] J. Sun and J. Chu, "Fourier series representation of the coupler curves of spatial linkages," Applied Mathematical Modelling, vol. 34, no. 5, pp. 1396-1403, 2010.

[14] J. Wu, Q. J. Ge, and F. Gao, "An efficient method for synthesizing crank-rocker mechanisms for generating low harmonic curves," in Proceedings of the 2009 ASME International Design 
Engineering Technical Conferences and Computers and Information in Engineering Conference, DETC2009, pp. 531-538, USA, September 2009.

[15] J. Chu, L. Wang, and C. Wu, "Relationship between coupler curve properties of a 4-bar linkage and its dimensional types," Science China Technological Sciences, vol. 47, no. 4, pp. 479-487, 2004.

[16] J. Xie and Y. Chen, "Application backpropagation neural network to synthesis of whole cycle motion generation mechanism," in Proceedings of the in Proceedings of the 12th IFToMM World Congress, Besançon, France, 2007.

[17] H. W. Guggenheimer, Differential Geometry and its Applications, Dover Publications, Inc., New York, NY, USA, 1977.

[18] S. Dikabar and T. S. Mruthyunjaya, "Synthesis of workspaces of planar manipulators with arbitrary topology using shape representation and simulated annealing," Mechanism and Machine Theory, vol. 34, no. 3, pp. 391-420, 1999.

[19] A. Vasiliu and B. Yannou, "Dimensional synthesis of planar mechanisms using neural networks: Application to path generator linkages," Mechanism and Machine Theory, vol. 36, no. 2, pp. 299-310, 2001.

[20] J. Wu, Q. J. Ge, F. Gao, and W. Z. Guo, "On the extension of a fourier descriptor based method for planar four-bar linkage synthesis for generation of open and closed paths," Journal of Mechanisms and Robotics, vol. 3, no. 3, Article ID 031002, 2011.

[21] F. T. S. Marín and A. P. González, "Global optimization in path synthesis based on design space reduction," Mechanism and Machine Theory, vol. 38, no. 6, pp. 579-594, 2003.

[22] G. Galán-Marín, F. J. Alonso, and J. M. Del Castillo, "Shape optimization for path synthesis of crank-rocker mechanisms using a wavelet-based neural network," Mechanism and Machine Theory, vol. 44, no. 6, pp. 1132-1143, 2009.

[23] L. P. J. Veelenturf, Analysis and Applications of Artifical Neural Networks, Hemel Hempstead, Hemel Hempstead, Hertfordshire, UK, 1995.

[24] T. J. Anastasio, Tutorial on Neural Systems Modeling, Sinauer Associates Inc, Sunderland, MA, USA, 2010.

[25] X. Li, J. Wu, and Q. J. Ge, “A fourier descriptor-based approach to design space decomposition for planar motion approximation," Journal of Mechanisms and Robotics, vol. 8, no. 6, Article ID 064501, 2016.

[26] W. H. Beyer, CRC Standard Mathematical Tables, CRC Press, Boca Raton, FL, USA, 28th edition, 1987. 


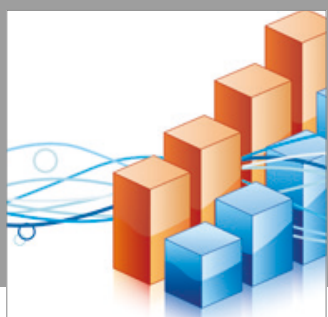

Advances in

Operations Research

vatersals

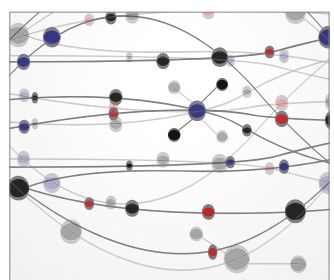

\section{The Scientific} World Journal
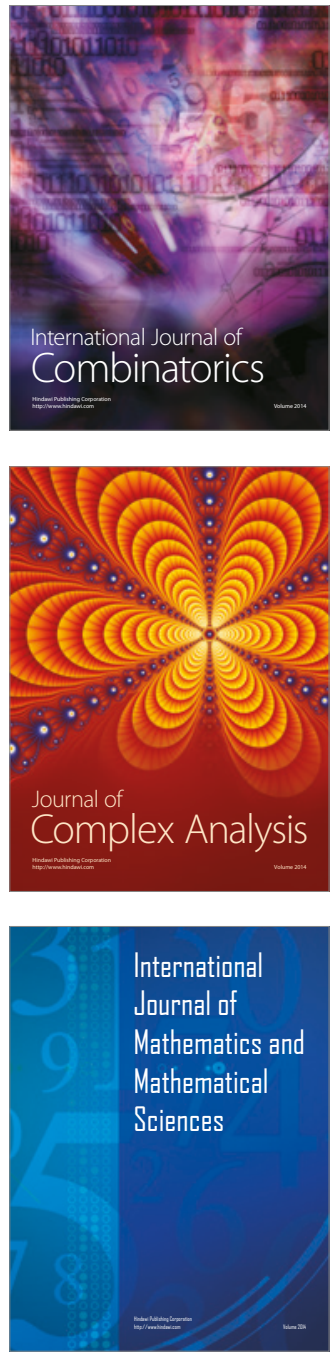
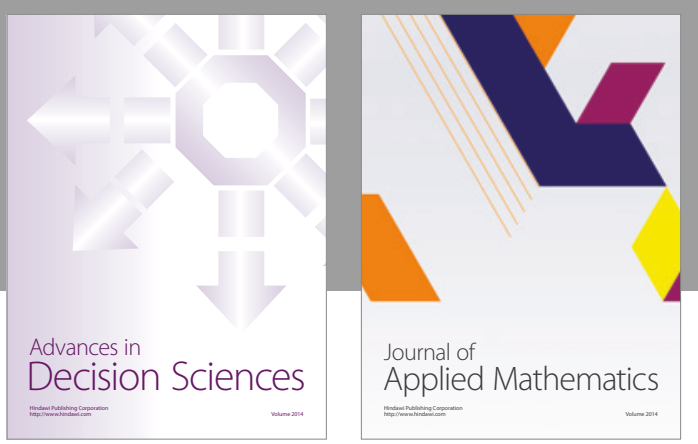

Algebra

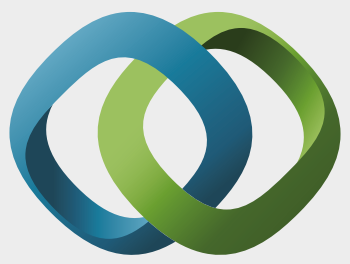

\section{Hindawi}

Submit your manuscripts at

https://www.hindawi.com
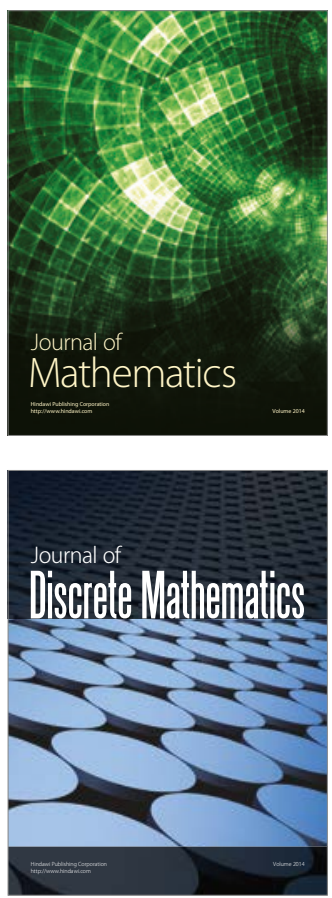

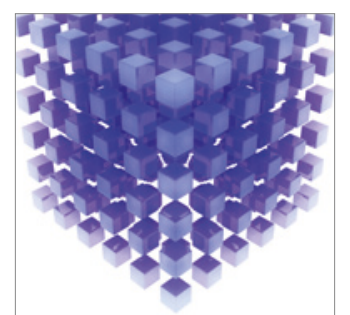

Mathematical Problems in Engineering
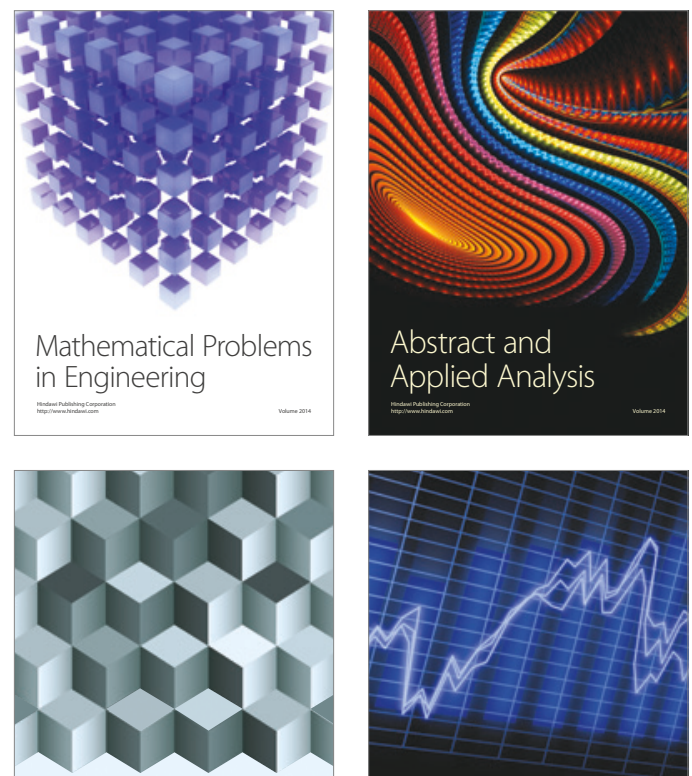

Journal of

Function Spaces

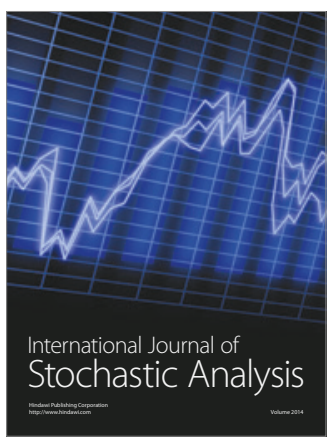

Probability and Statistics
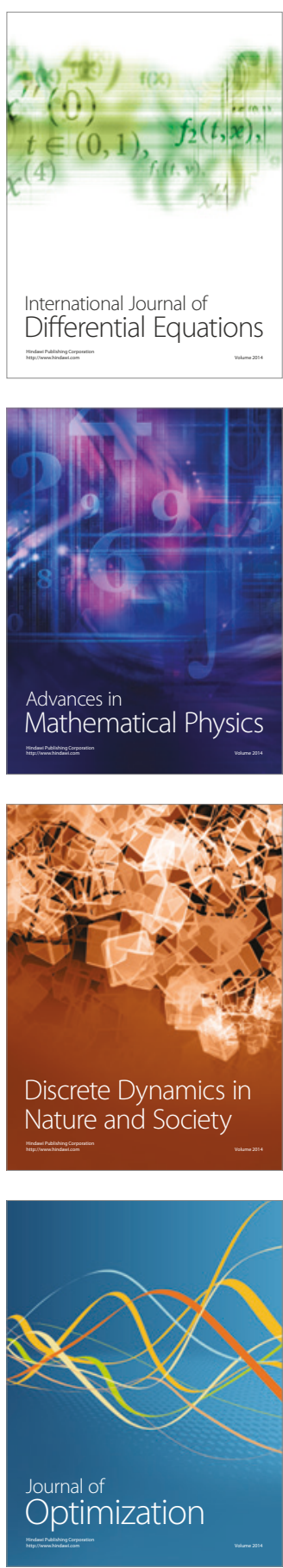